\title{
Direct and Divergent Solid-Phase Synthesis of Azobenzene and Spiropyran Derivatives
}

\author{
Ravichandran H. Kollarigowda, ${ }^{1,2,4}$, Paul V. Braun*1,2,3,4 \\ 1 Beckman Institute for Advanced Science and Technology, University of Illinois at Urbana-Champaign, \\ Urbana, IL 61801 \\ 2 Department of Materials Science and Engineering, University of Illinois at Urbana-Champaign, Urbana, IL \\ 61801 \\ 3 Department of Chemistry, University of Illinois at Urbana-Champaign, Urbana, IL 61801 \\ 4 Materials Research Laboratory, University of Illinois at Urbana-Champaign, Urbana, IL 61801.
}

Corresponding Author: pbraun@illinois.edu 


\section{SUPPORTING INFORMATION}

\section{Table of Contents}

1. Spiropyrans synthesis scheme................................. S3

2. $\quad$ Kaiser test....................................................... S4

3. Colorimetric Kaiser tests....................................... S4

4. Figure S1. Absorption spectrum of Fmoc- aminobenzoic acid-resin.... S4

5. $\quad$ Eq S(1) Fmoc substitution calculation.............................. S5

6. NMR and Mass spectra of azobenzenes............................. S6-S17

7. NMR and Mass spectra of spiropyrans........................... S18-S31

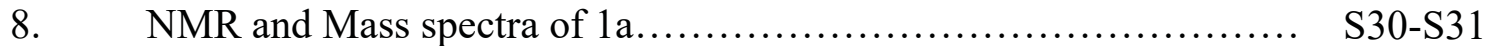

9. $\quad$ NMR and Mass spectra of B1 ................................... S323

10. HPLC of azobenzenes and spiropyrans.......................... S34-S45 


\section{Spiropyran synthesis scheme}

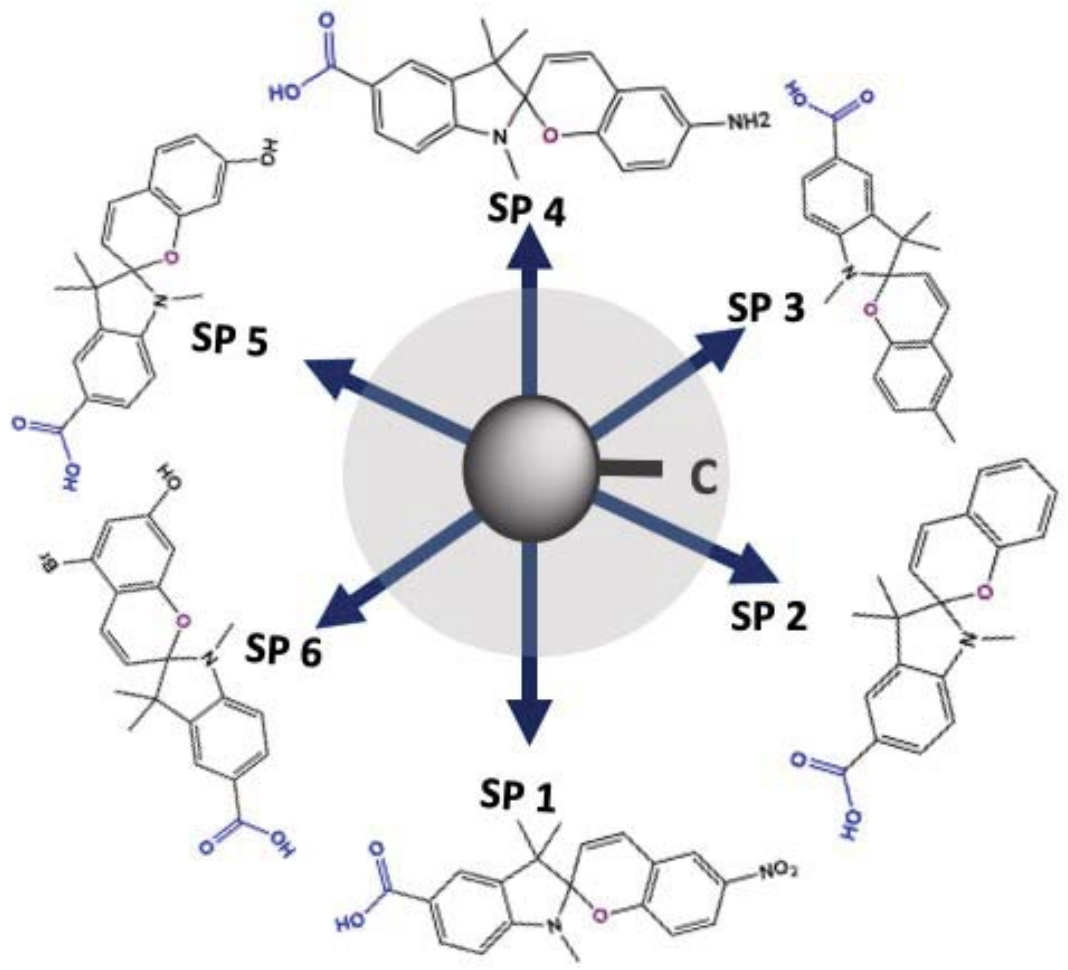

Scheme S1. Synthesized spiropyrans 
Kaiser test. Remove a few resin beads from the reaction vessel and wash 3 times with ethanol. Transfer beads into a small glass tube and add three drops of each solution. Mix well and heat the tube at $80^{\circ} \mathrm{C}$ for 10 minutes. The resin beads and the solution turn dark blue when primary amines are present. The resin beads remain their color and the solution stay yellow when no free primary amine is present (expected result after successful coupling). A re-coupling step is necessary when a slight blue color is detected in the solution and/or on beads.

Colorimetric Kaiser tests; Briefly, the Kaiser reagent (ninhydrin) is added to a few resin beads and heated for 10 minutes at $80^{\circ} \mathrm{C}$. If unreacted amine remains, the Kaiser reagent changes in color from yellow to violet. If the color does not change, it means that the resin has completely reacted with block $\mathbf{B}$.

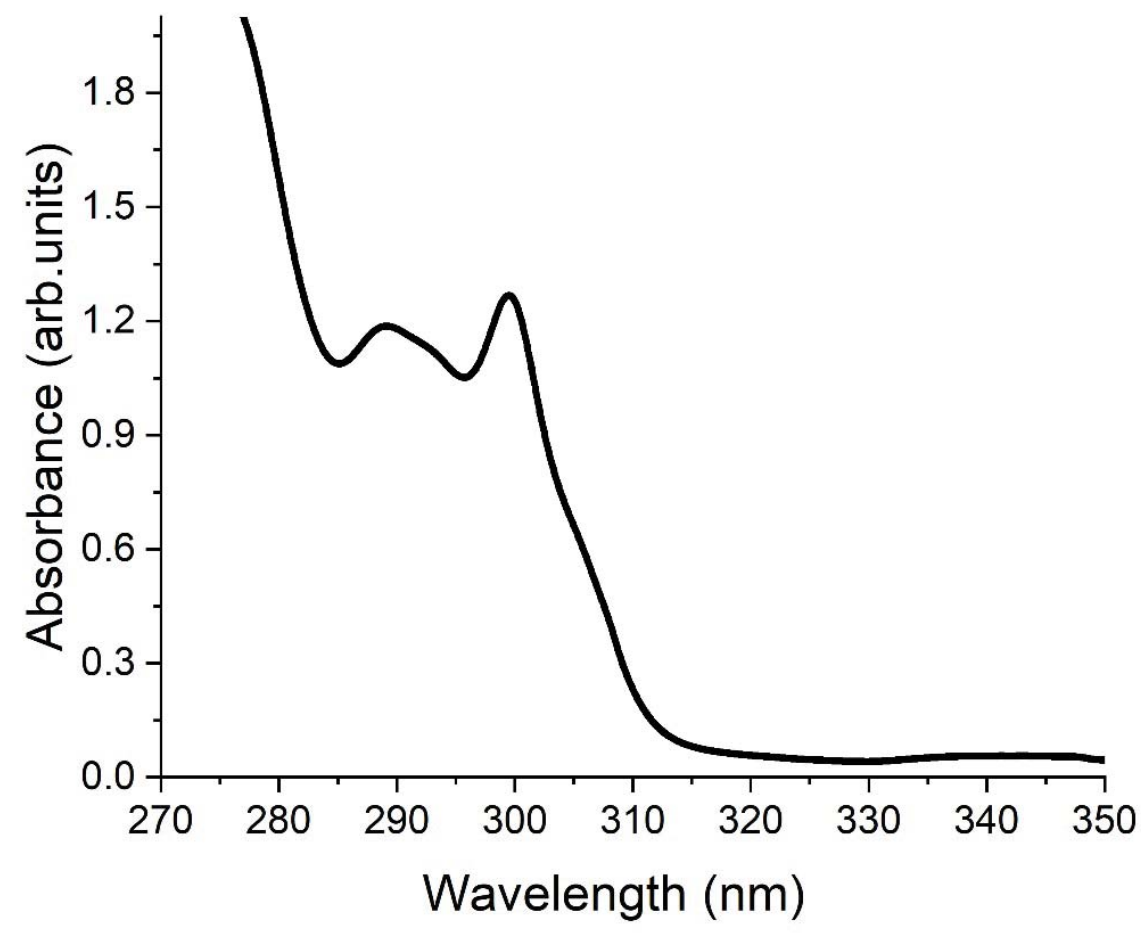

Figure S1. Absorption spectrum of Fmoc- aminobenzoic acid-resin -piperidine in 20\% (v/v) piperidine in DMF between 270 and $310 \mathrm{~nm}$. 
The Fmoc substitution may be calculated with the following formula using the maximum at $289.8 \mathrm{~nm}$ (for the maximum at $301.0 \mathrm{~nm}$, the calculation is performed analogously):

$\mathrm{SFmoc}\left[\mathrm{mmol} \mathrm{g}^{-1}\right]=\frac{\mathrm{E} 289.8 \mathrm{~nm} \times \mathrm{V} \times \mathrm{D}}{\varepsilon 289.8 \mathrm{~nm} \times \mathrm{mResin} \times \mathrm{l}} \quad-\mathrm{Eq} \mathrm{S}(1)$

where:

$\mathrm{S}_{\mathrm{Fmoc}}=$ Fmoc substitution $\left[\mathrm{mmol} \mathrm{g}^{-1}\right]$

$\varepsilon 289.8 \mathrm{~nm}=$ Molar absorption coefficient at $289.8 \mathrm{~nm}$

$\mathrm{E}_{289.8 \mathrm{~nm}}=$ Absorption of the sample solution at $289.8 \mathrm{~nm}$

$\mathrm{mResin}=$ Sample weight of the resin $[\mathrm{mg}] \mathrm{mmol} \mathrm{mol}^{-1} \mathrm{mg} \mathrm{g}^{-1}=$ Factor for conversion of mol to $\mathrm{mmol}$ and $\mathrm{mg}^{-1}$ to $\mathrm{g}^{-1}: 1000$ [mol to $\left.\mathrm{mmol}\right] 1000\left[\mathrm{mg}^{-1}\right.$ to $\mathrm{g}^{-1}$ ]

$\mathrm{V}=$ Sample volume

$1=$ Optical path length of the cell in $\mathrm{cm}(\mathrm{e} . \mathrm{g} .1 \mathrm{~cm})$

$\mathrm{D}=$ Dilution factor. 


\section{NMR and Mass spectra of azobenzenes}

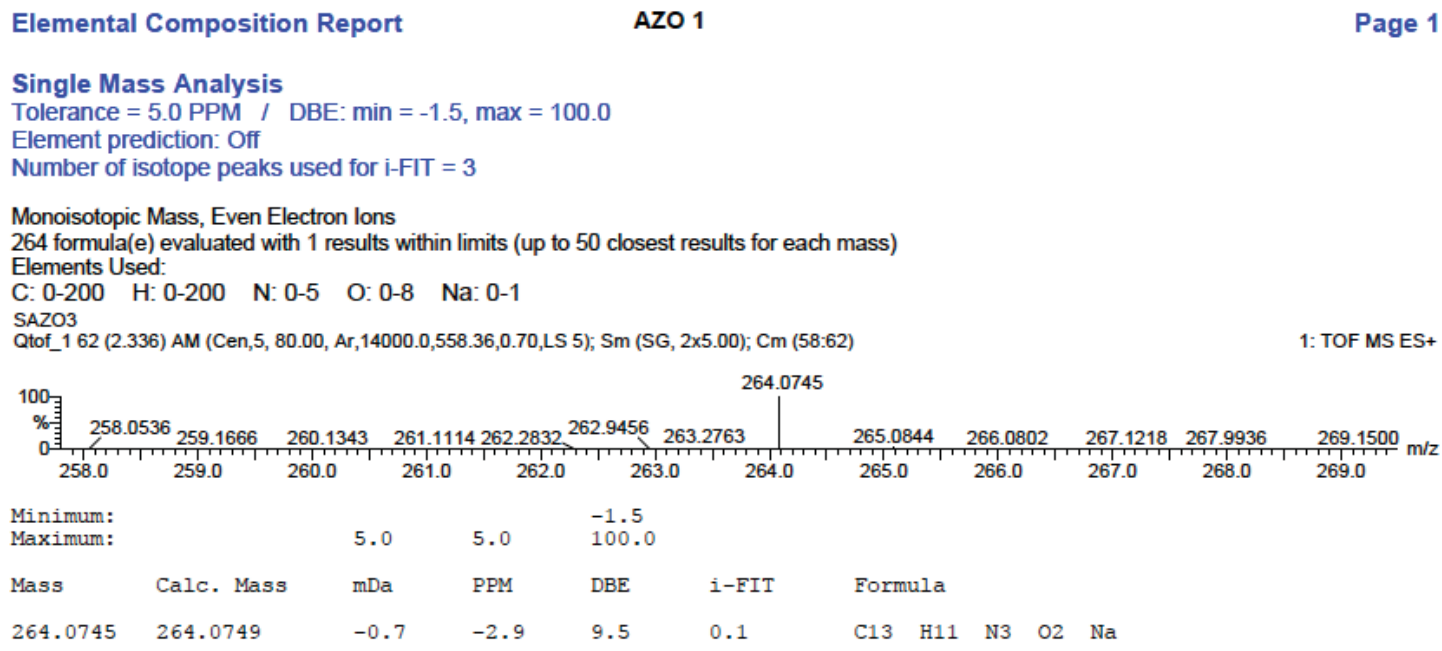


AZO 1, 'H NMR, $500 \mathrm{MHz}$, DMSO

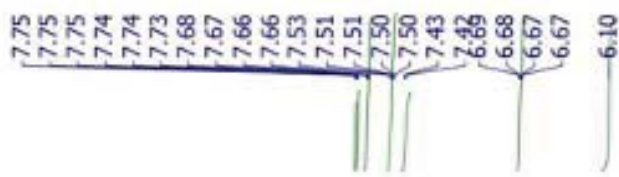<smiles>Nc1ccc(/N=N/c2ccc(C(=O)O)cc2)cc1</smiles>

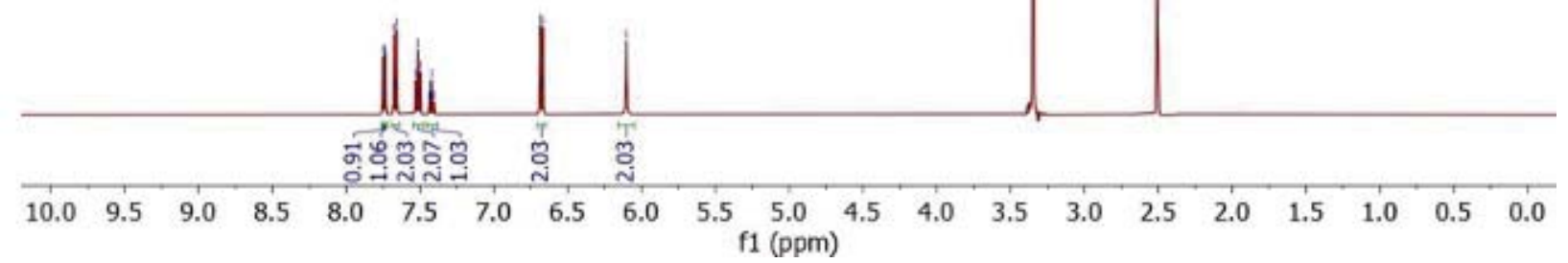

${ }^{13} \mathrm{C}\{1 \mathrm{H}\}$ for proton-decoupled carbon data.

AZO $1,{ }^{13} \mathrm{C}$ NMR, $126 \mathrm{MHz}$, DMSO

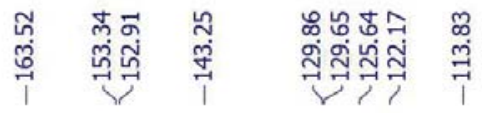
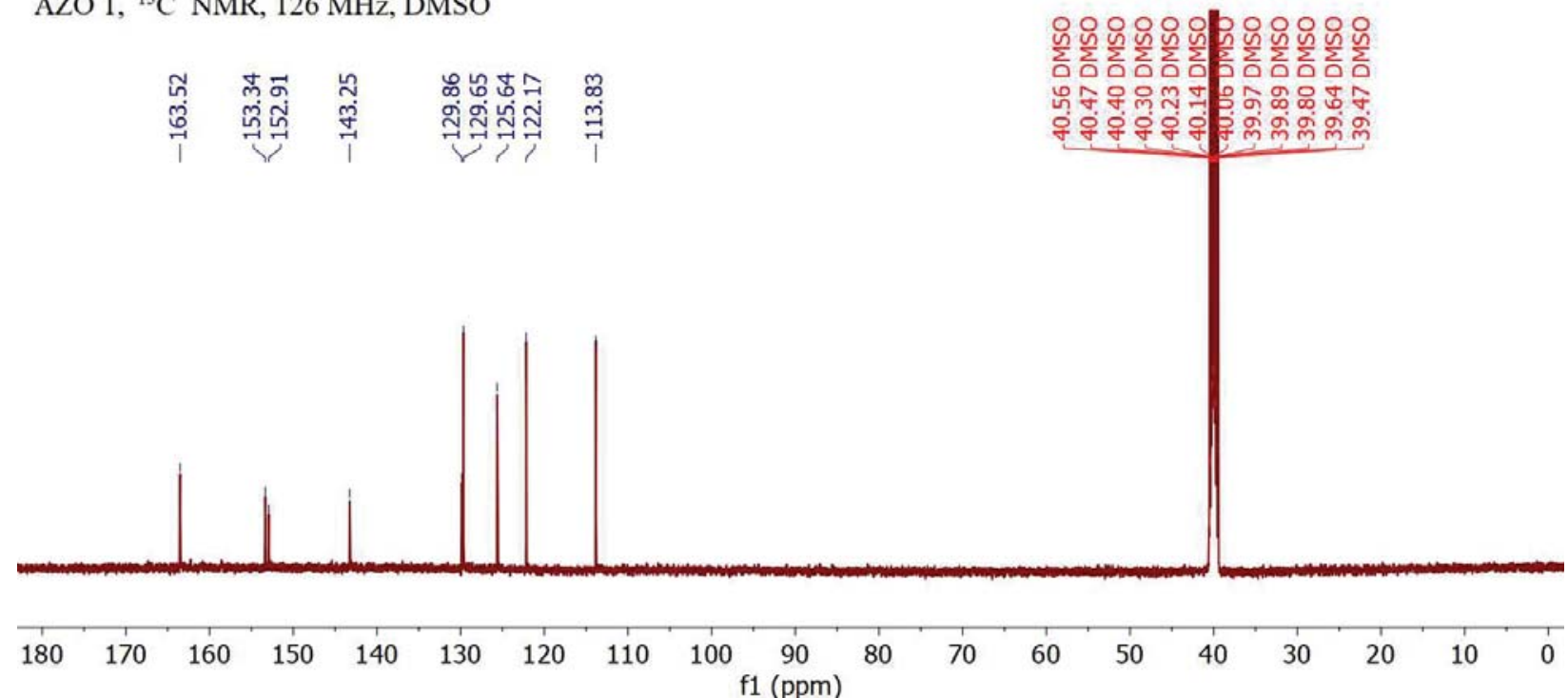
Single Mass Analysis

Tolerance $=5.0$ PPM I DBE: $\min =-1.5, \max =100.0$

Element prediction: Off

Number of isotope peaks used for i-FIT $=3$

Monoisotopic Mass, Even Electron lons

265 formula(e) evaluated with 1 results within limits (up to 50 closest results for each mass)

Elements Used:

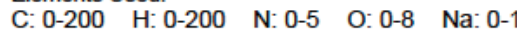

AZO 2 Qtof_2 67 (2.538) AM (Cen,5, 80.00, Ar, 14000.0,558.36,0.70,LS 5); Sm (SG, 2×5.00); Cm (64:67)

1: TOF MS ES+

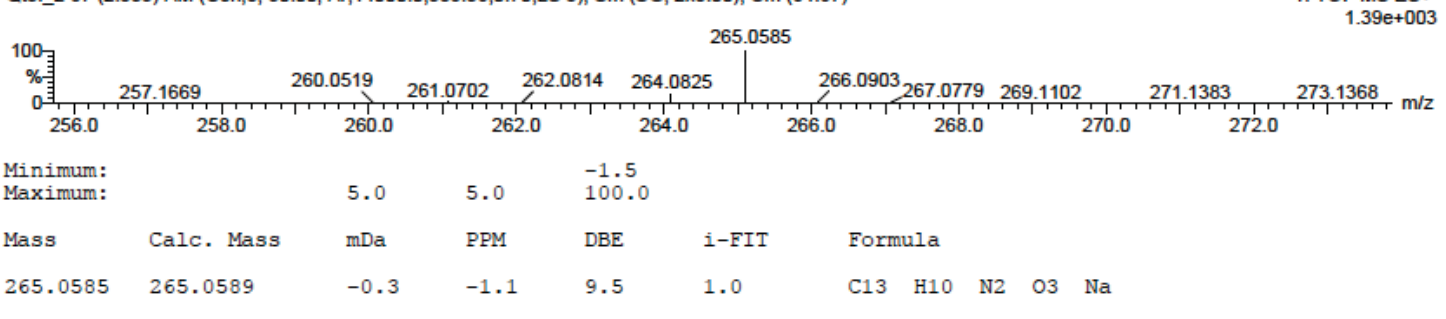


AZO 2,1H NMR, $500 \mathrm{MHz}$, DMSO

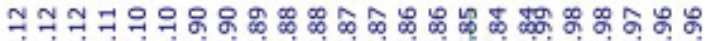

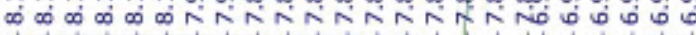
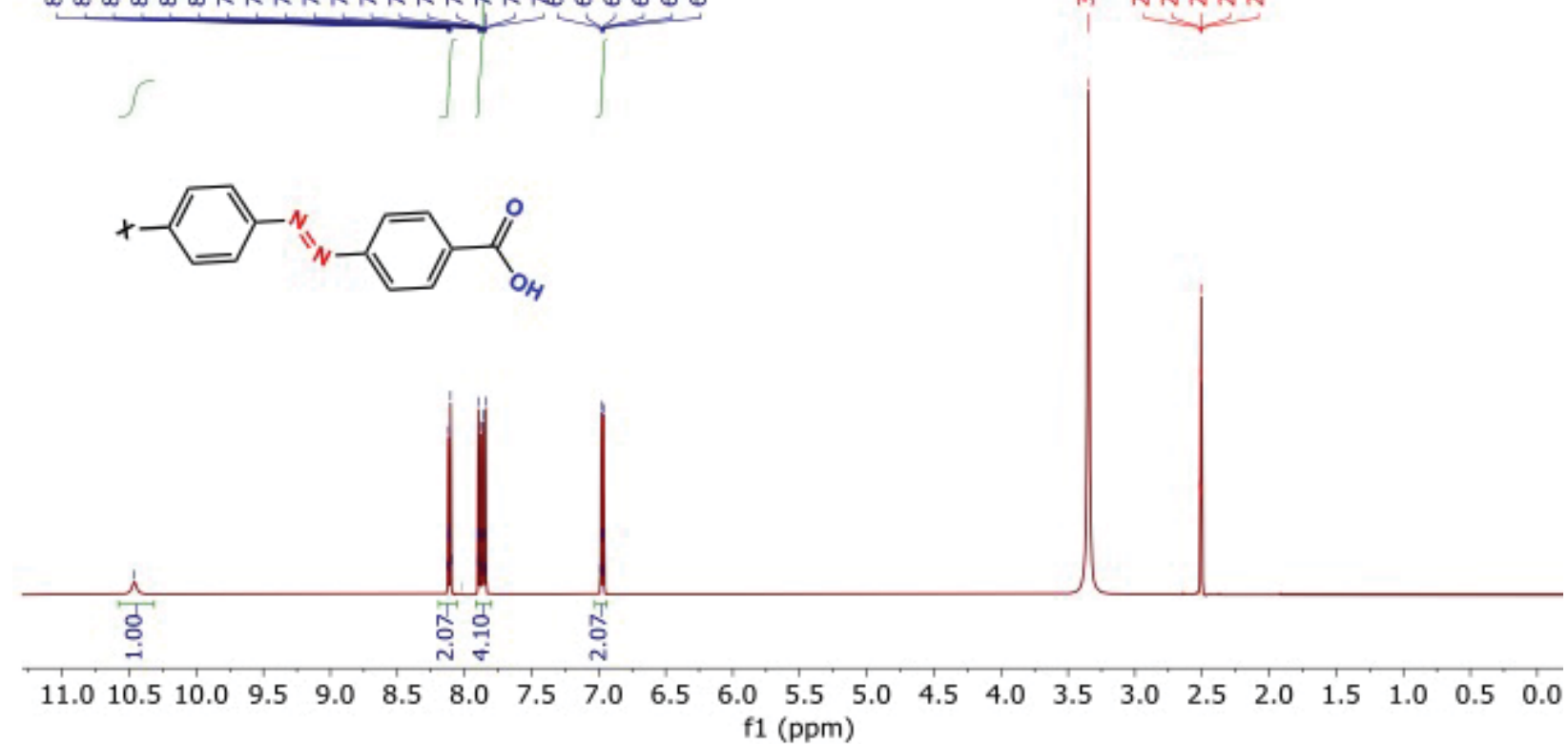

${ }^{13} \mathrm{C}\{1 \mathrm{H}\}$ for proton-decoupled carbon data.

AZO $2,{ }^{13} \mathrm{C}$ NMR, $126 \mathrm{MHz}$, DMSO

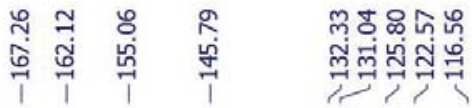
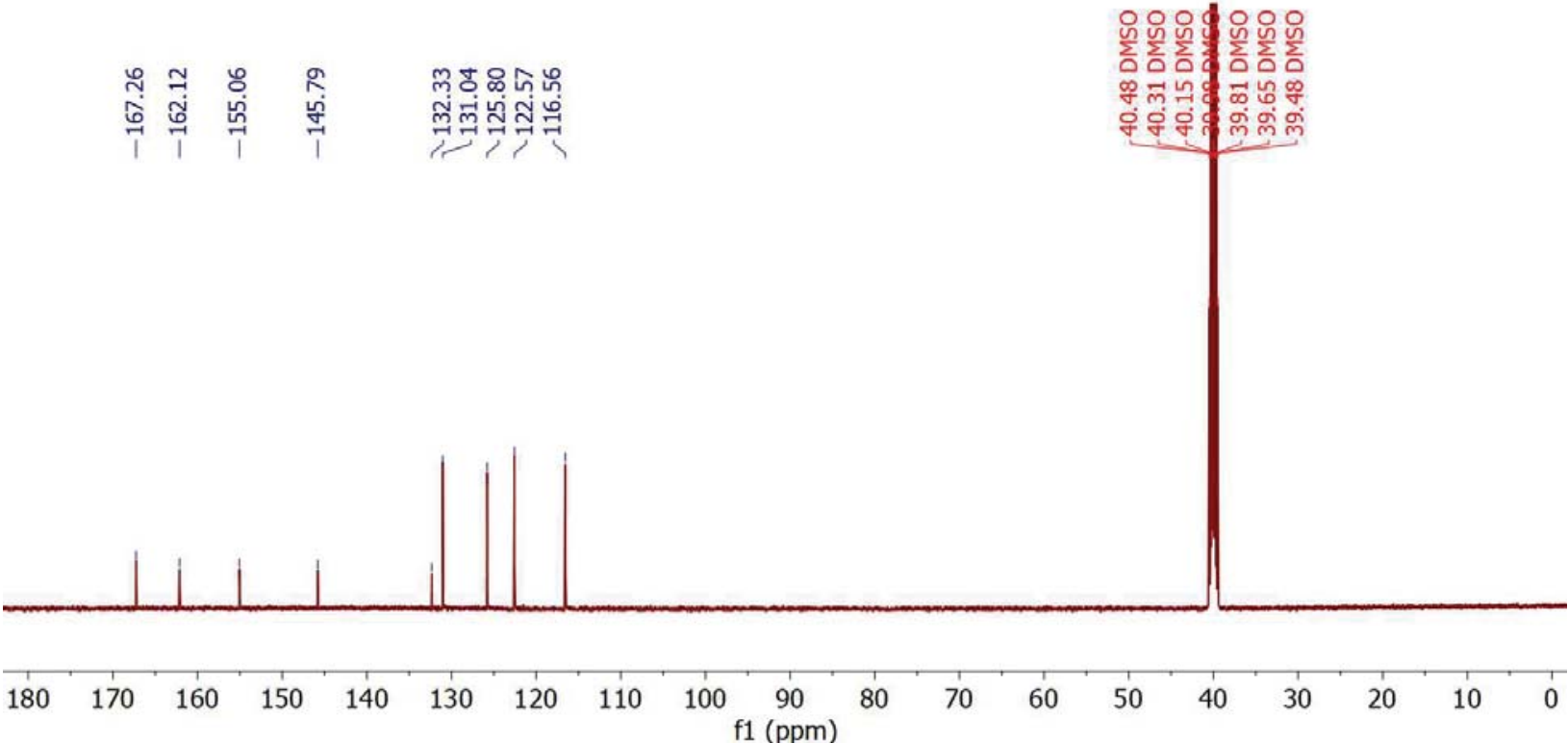
Single Mass Analysis

Tolerance $=5.0$ PPM / DBE: $\min =-1.5, \max =100.0$

Element prediction: Off

Number of isotope peaks used for i-FIT = 3

Monoisotopic Mass, Even Electron lons

320 formula(e) evaluated with 1 results within limits (up to 50 closest results for each mass)

Elements Used:

$\begin{array}{lllll}\text { C: } 0-200 & \mathrm{H}: 0-200 & \mathrm{~N}: 0-5 & \mathrm{O}: 0-6 & \mathrm{Na}: 0-1 \\ \mathrm{Br}: \text { 1-1 }\end{array}$

AZO 3 Qtof_363 (2.370) AM (Cen,5, 80.00, Ar, 14000.0,558.36,0.70,LS 5); Sm (SG, 2x5.00); Cm (60:63)

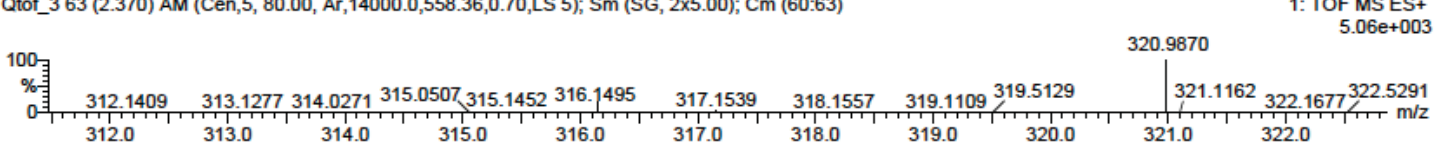

Minimum:

Maximum:

$\begin{array}{lll}5.0 & 5.0 \quad 100.0\end{array}$

Mass Calc. Mass mDa PPM DBE i-FIT Formula

$\begin{array}{lllllllllll}320.9870 & 320.9875 & -0.5 & -1.6 & 9.5 & 7.4 & \text { C13 } & \text { H10 } & \text { N2 } & \text { O3 } & \text { Br }\end{array}$ 


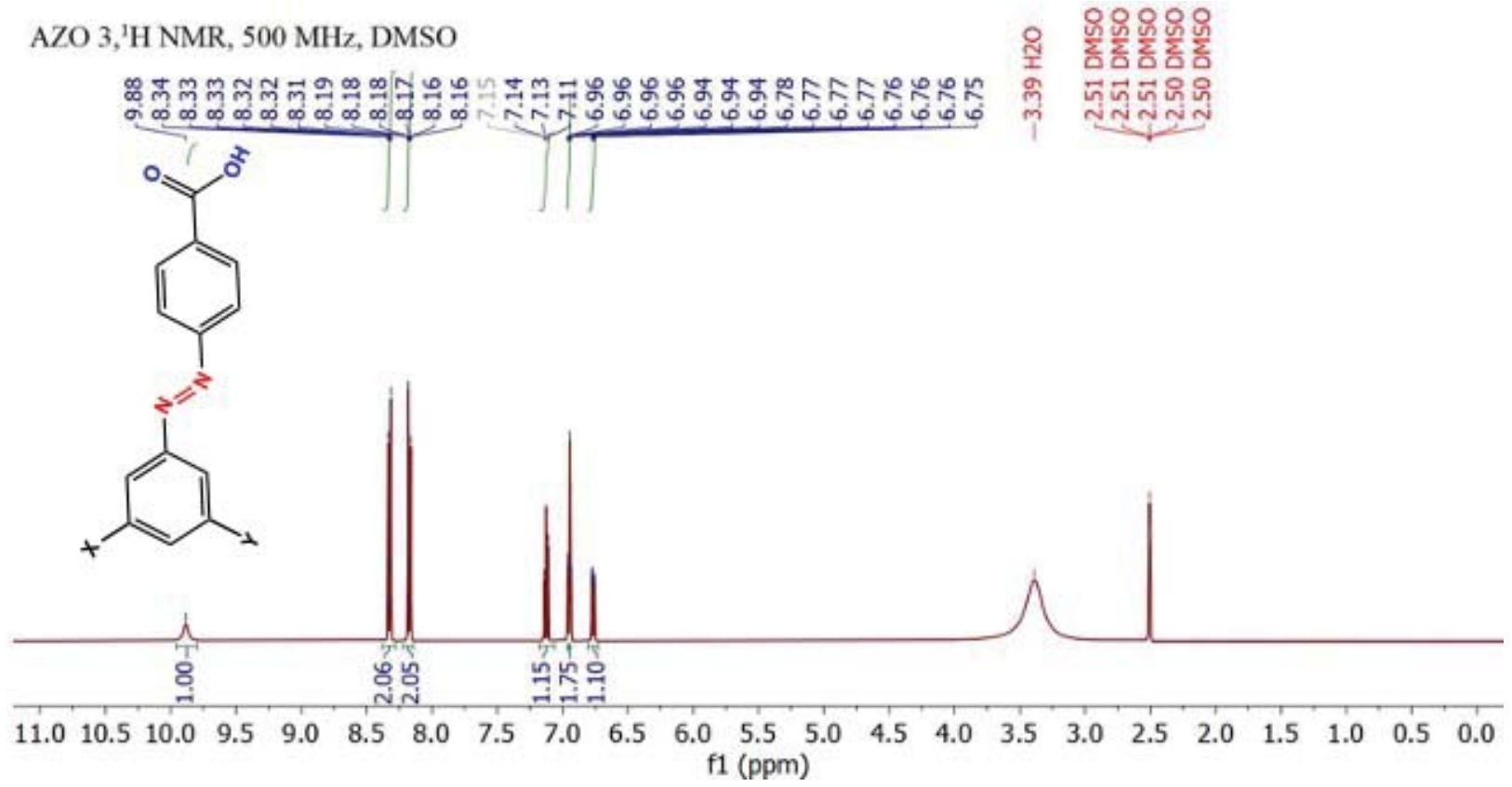

${ }^{13} \mathrm{C}\{1 \mathrm{H}\}$ for proton-decoupled carbon data.

AZO $3,{ }^{13} \mathrm{C}$ NMR, $126 \mathrm{MHz}$, DMSO

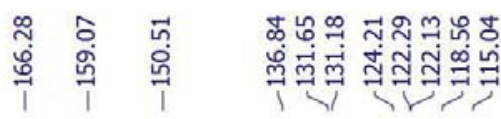
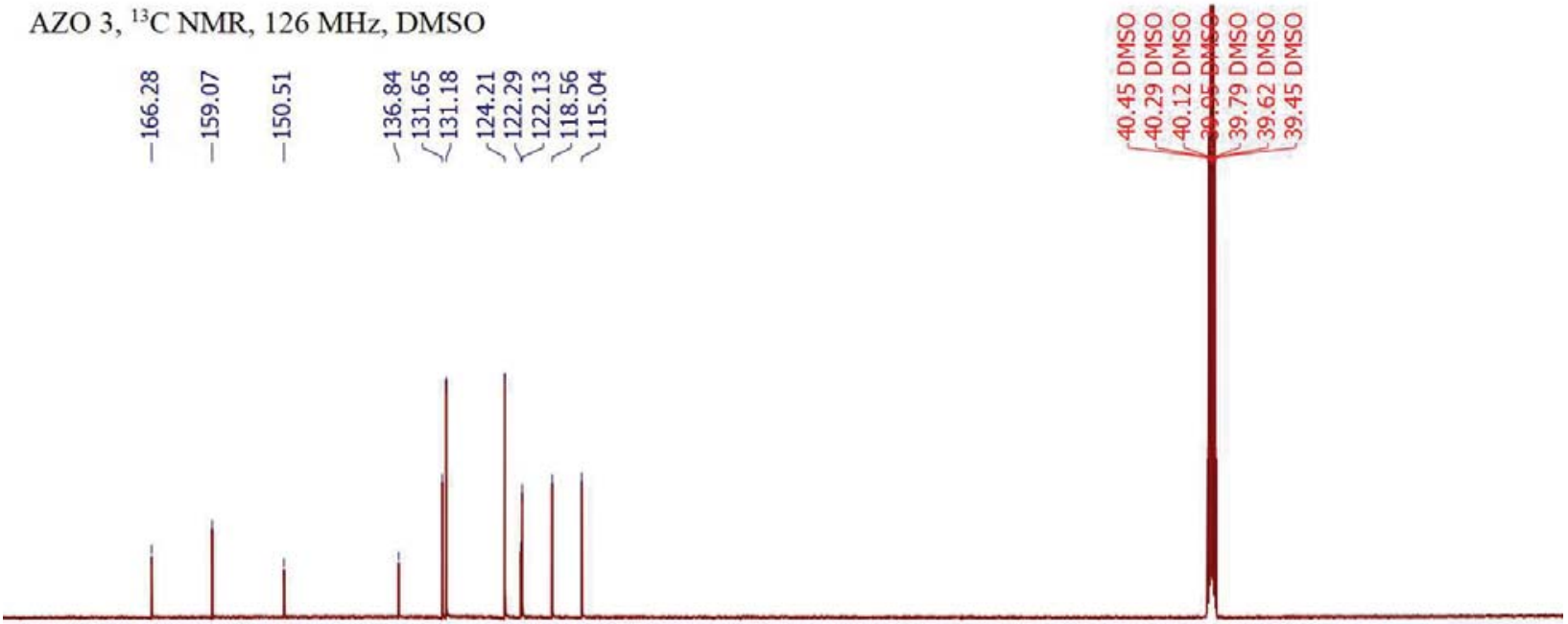

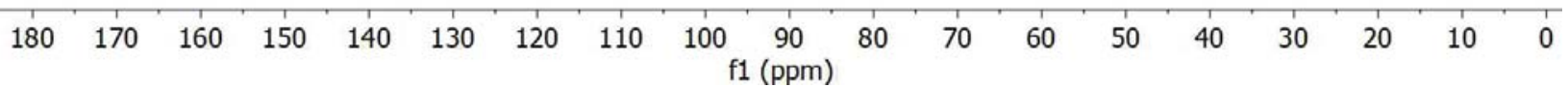


Single Mass Analysis

Tolerance $=5.0$ PPM / DBE: $\min =-1.5, \max =100.0$

Element prediction: Off

Number of isotope peaks used for i-FIT $=3$

Monoisotopic Mass, Even Electron lons

309 formula(e) evaluated with 1 results within limits (up to 50 closest results for each mass)

Elements Used:

$\begin{array}{lllll}\text { C: } 0-200 & \mathrm{H}: 0-200 & \mathrm{~N}: 0-5 & \mathrm{O}: 0-8 & \mathrm{Na}: 0-1\end{array}$

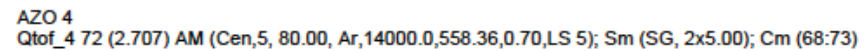

1: TOF MS ES+ 309.0849

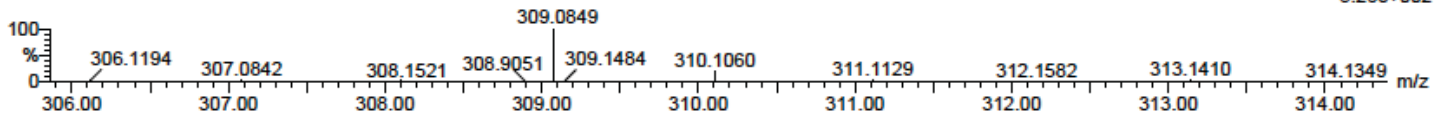

$\begin{array}{llll}\text { Minimum: } & & & \\ \text { Maximum: } & 5.0 & 5.0 & 100.0\end{array}$

Mass Calc. Mass mDa PPM DBE i-FIT Formula

$\begin{array}{lllllllllll}309.0849 & 309.0851 & 0.3 & 1.2 & 11.5 & 1.6 & \text { C15 } & \text { H14 } & \text { N2 } & 04 & \text { Na }\end{array}$ 


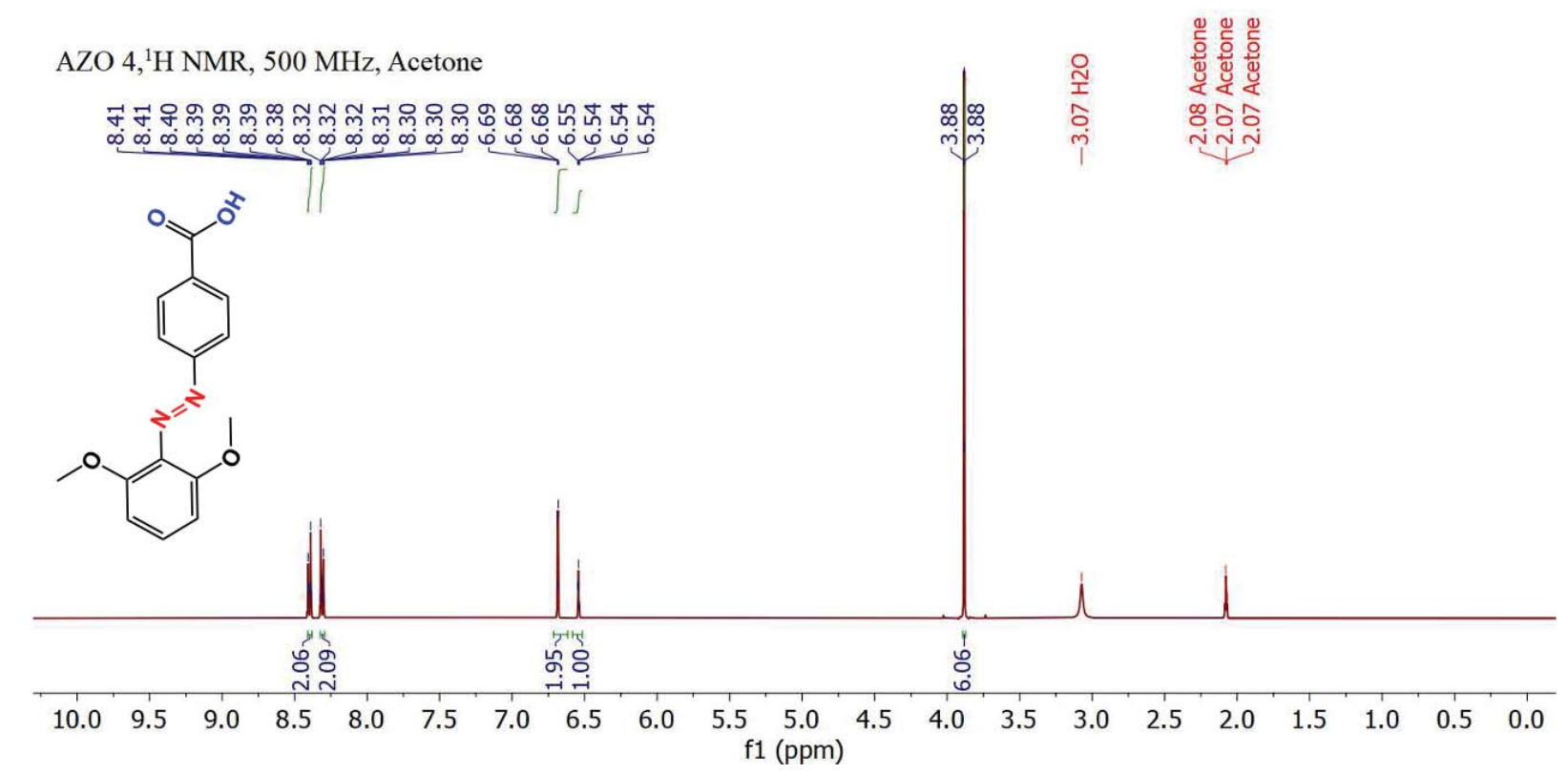

${ }^{13} \mathrm{C}\{1 \mathrm{H}\}$ for proton-decoupled carbon data.

AZO $4,{ }^{13} \mathrm{C}$ NMR, $126 \mathrm{MHz}$, DMSO

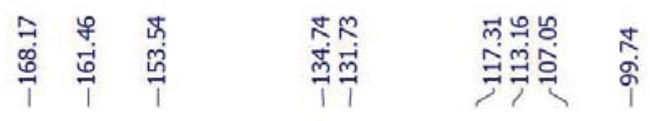

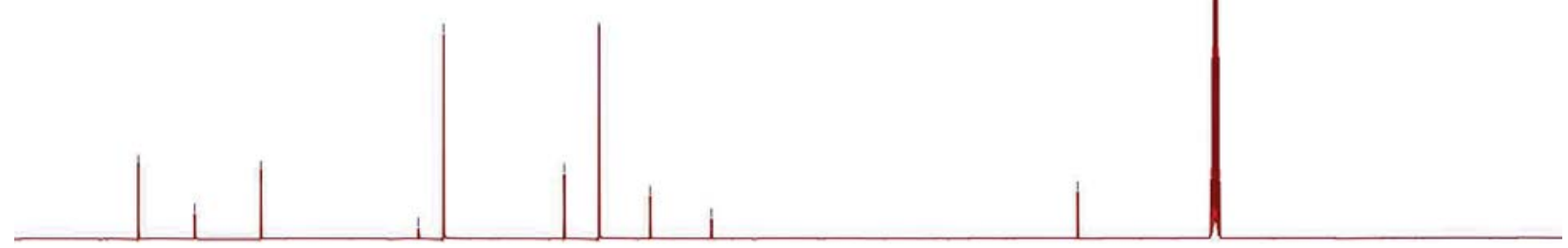

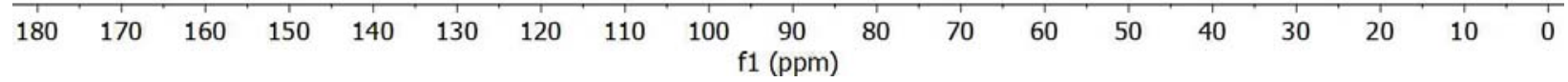


Single Mass Analysis

Tolerance $=5.0$ PPM I DBE: $\min =-1.5, \max =100.0$

Element prediction: Off

Number of isotope peaks used for $\mathrm{i}-\mathrm{FIT}=3$

Monoisotopic Mass, Even Electron lons

379 formula(e) evaluated with 1 results within limits (up to 50 closest results for each mass)

Elements Used:

$\begin{array}{lllll}\text { C: } 0-200 & \text { H: } 0-200 & \text { N: } 0-5 & \text { O: } 0-6 & \text { Na: } 0-1\end{array}$

AZO 5 Qtof_5 68 (2.572) AM (Cen,5, 80.00, Ar, 14000.0,558.36,0.70,LS 5); Sm (SG, 2×5.00); Cm (68:79)

1. TOF MS ES+

$$
379.1443
$$

$3.16 \mathrm{e}+002$

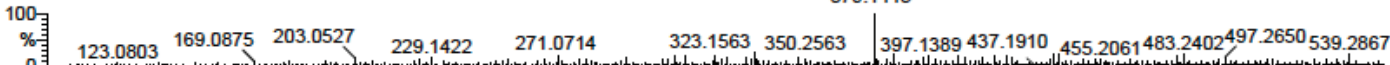

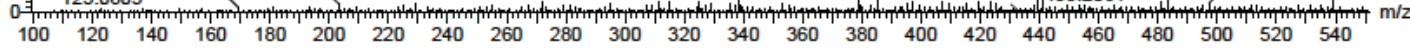

Minimum

Maximum:

-1.5
100.0

Mass

Calc. Mass

5.0

5.0

379.1443

379.1447

$\begin{array}{ll}-0.5 & -1.8\end{array}$

$\begin{array}{ll}10.5 & 23.8\end{array}$

i-FIT

Formula

C25 H19 N2 O2 
AZO 5, 'H NMR, $500 \mathrm{MHz}$, Acetone

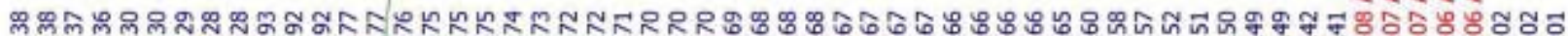
m

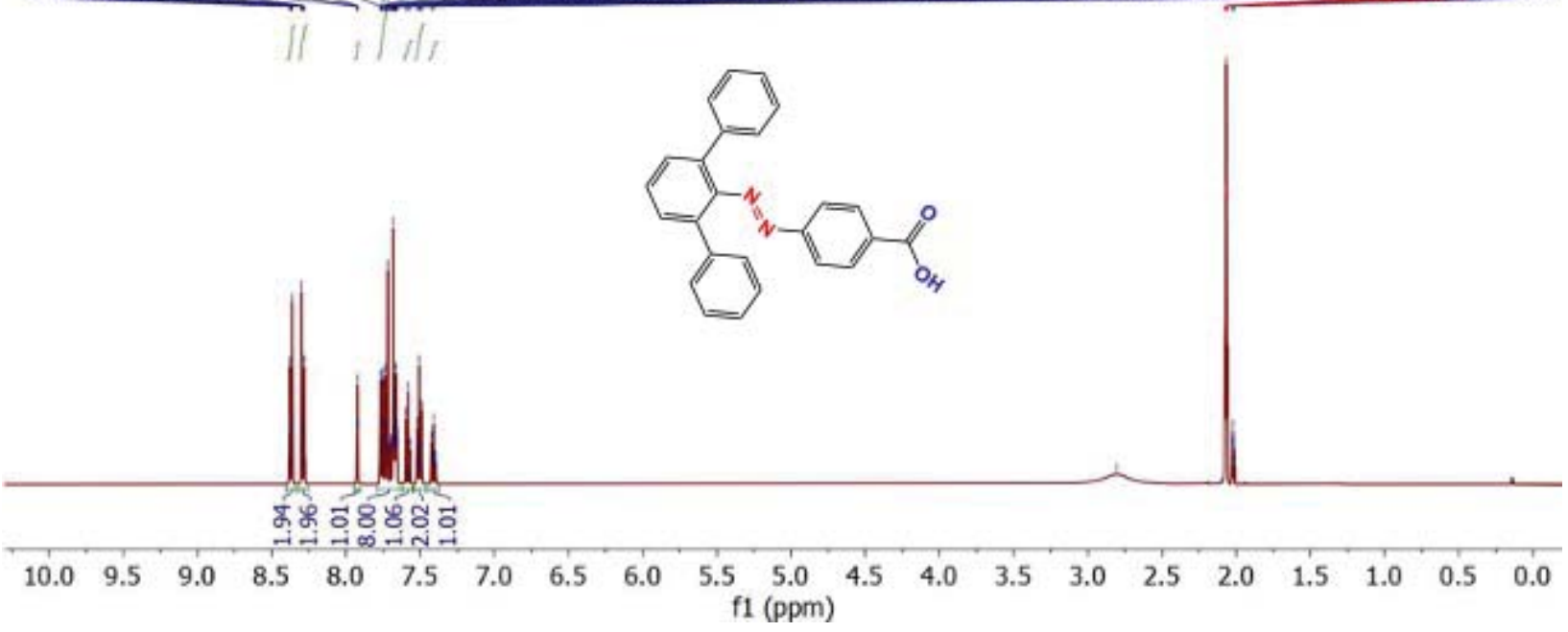

${ }^{13} \mathrm{C}\{1 \mathrm{H}\}$ for proton-decoupled carbon data.

AZO $5,{ }^{13} \mathrm{C}$ NMR, $126 \mathrm{MHz}$, Acetone
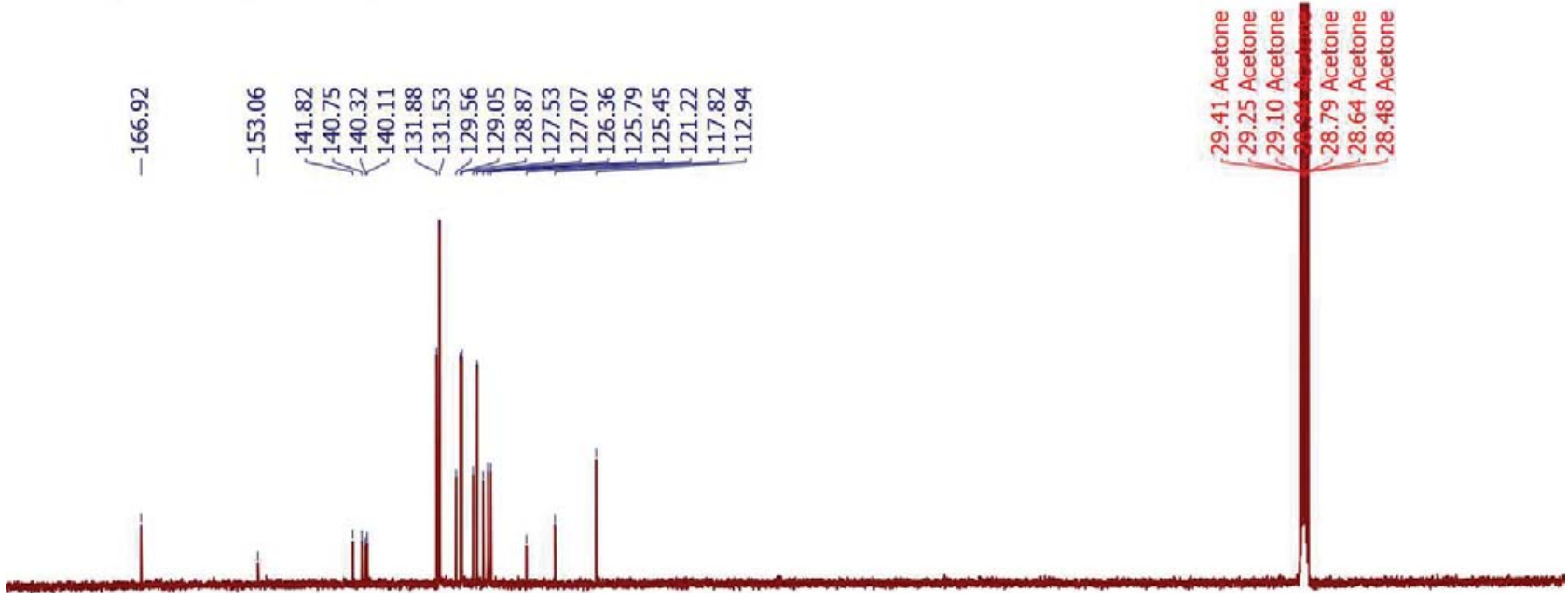

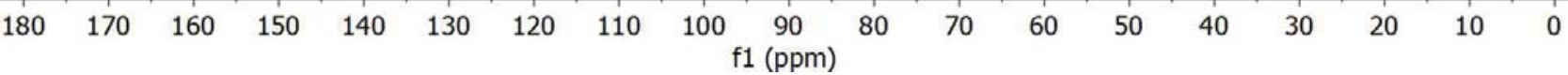


Single Mass Analysis

Tolerance $=5.0$ PPM / DBE: $\min =-1.5, \max =100.0$

Element prediction: Off

Number of isotope peaks used for i-FIT = 3

Monoisotopic Mass, Even Electron lons

299 formula(e) evaluated with 1 results within limits (up to 50 closest results for each mass)

Elements Used:

$\begin{array}{lllll}\text { C: } 0-200 & \mathrm{H}: 0-200 & \mathrm{~N}: 0-5 & \mathrm{O}: 0-8 & \mathrm{Na}: 0-1\end{array}$

AZO 6
Qtof_71889 62 (2.336) AM (Cen,5, 80.00, Ar, 14000.0,558.36,0.70,LS 5); Sm (SG, 2x5.00); Cm (59:62)

Qtof_71889 62 (2.336) AM (Cen, 5, 80.00, Ar, 14000.0,558.36,0.70,LS 5); Sm (SG, 2x5.00); Cm (59:62) $6.20 \mathrm{e}+003$

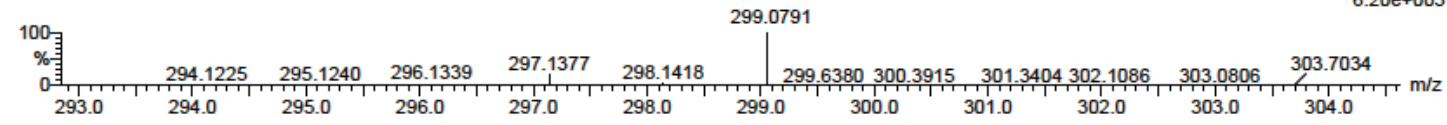

Minimum:

Maximum:

5.0

5.0

$-1.5$

Mass

Calc. Mass

$\mathrm{mDa}$

PPM

DBE

i-FIT

Formula

$299.0791 \quad 299.0796$

$\begin{array}{lll}-0.5 & -2.2 & 8.5\end{array}$

5.1

$\begin{array}{lllll}\mathrm{C} 17 & \mathrm{H} 12 & \mathrm{~N} 2 & \mathrm{O} 2 & \mathrm{Na}\end{array}$ 
AZO $6,{ }^{1} \mathrm{H}$ NMR, $500 \mathrm{MHz}$, DMSO

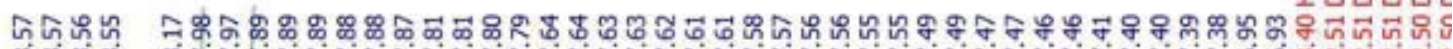

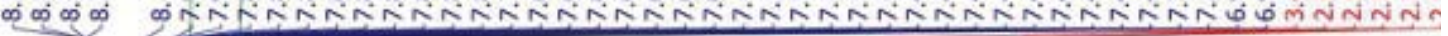
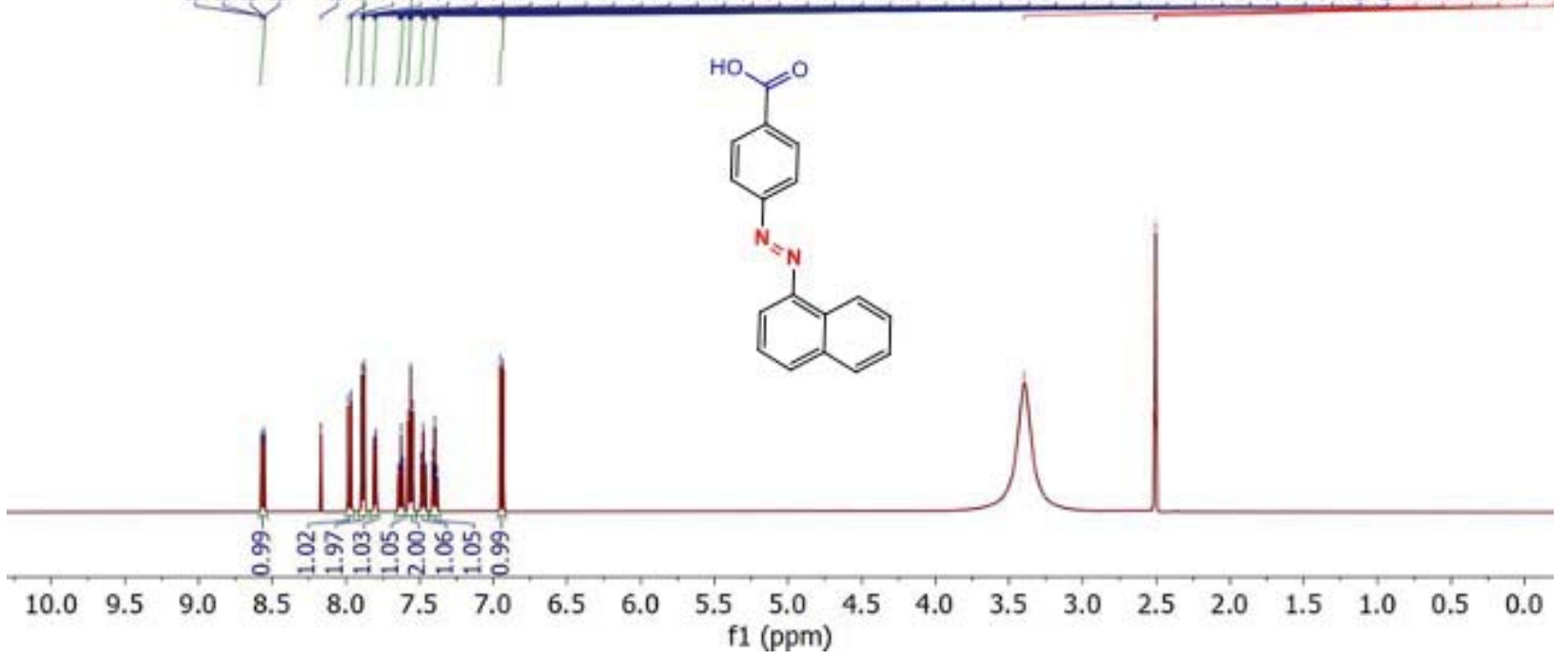

${ }^{13} \mathrm{C}\{1 \mathrm{H}\}$ for proton-decoupled carbon data.

AZO $6,{ }^{13} \mathrm{C}$ NMR, $126 \mathrm{MHz}$, DMSO
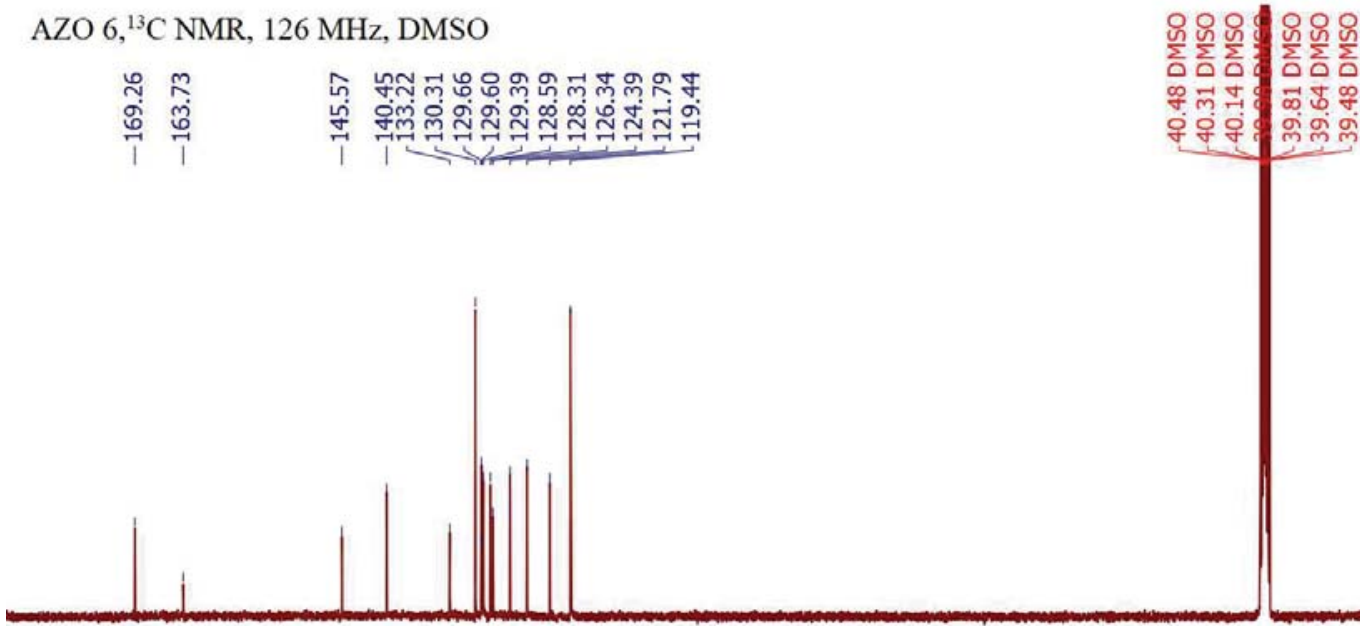

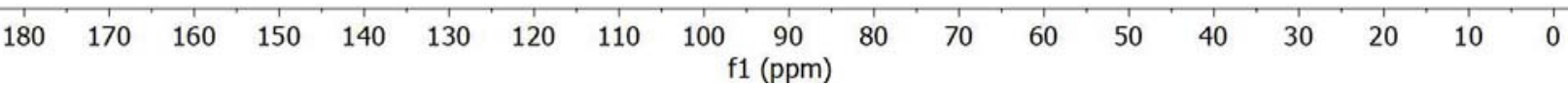




\section{NMR and Mass spectra of spiropyrans}

Single Mass Analysis

Tolerance $=5.0$ PPM / DBE: $\min =-1.5, \max =100.0$

Element prediction: Off

Number of isotope peaks used for i-FIT $=3$

Monoisotopic Mass, Even Electron lons

367 formula(e) evaluated with 1 results within limits (up to 50 closest results for each mass)

Elements Used:

$\begin{array}{lllll}\text { C: } 0-200 & \text { H: } 0-200 & \text { N: } 0-5 & \text { O: 0-6 Na: 0-1 }\end{array}$

SP 1 Qtof_7 74 (2.808) AM (Cen,5, 80.00, Ar, 14000.0,558.36,0.70,LS 5); Sm (SG, 2x5.00); Cm (74:77)

1: TOF MS ES+

$7.55 \mathrm{e}+003$

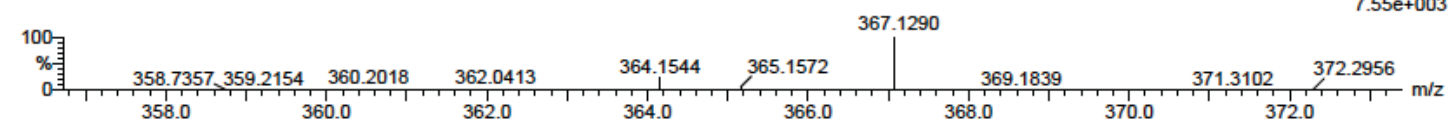

Minimum:

Maximum:

362.0

$-1.5$

Mass Calc. Mass DPM

$\begin{array}{llllllllll}367.1290 & 367.1294 & -0.2 & -0.6 & 11.5 & 4.2 & \text { C20 } & \text { H19 } & \text { N2 } & 05\end{array}$




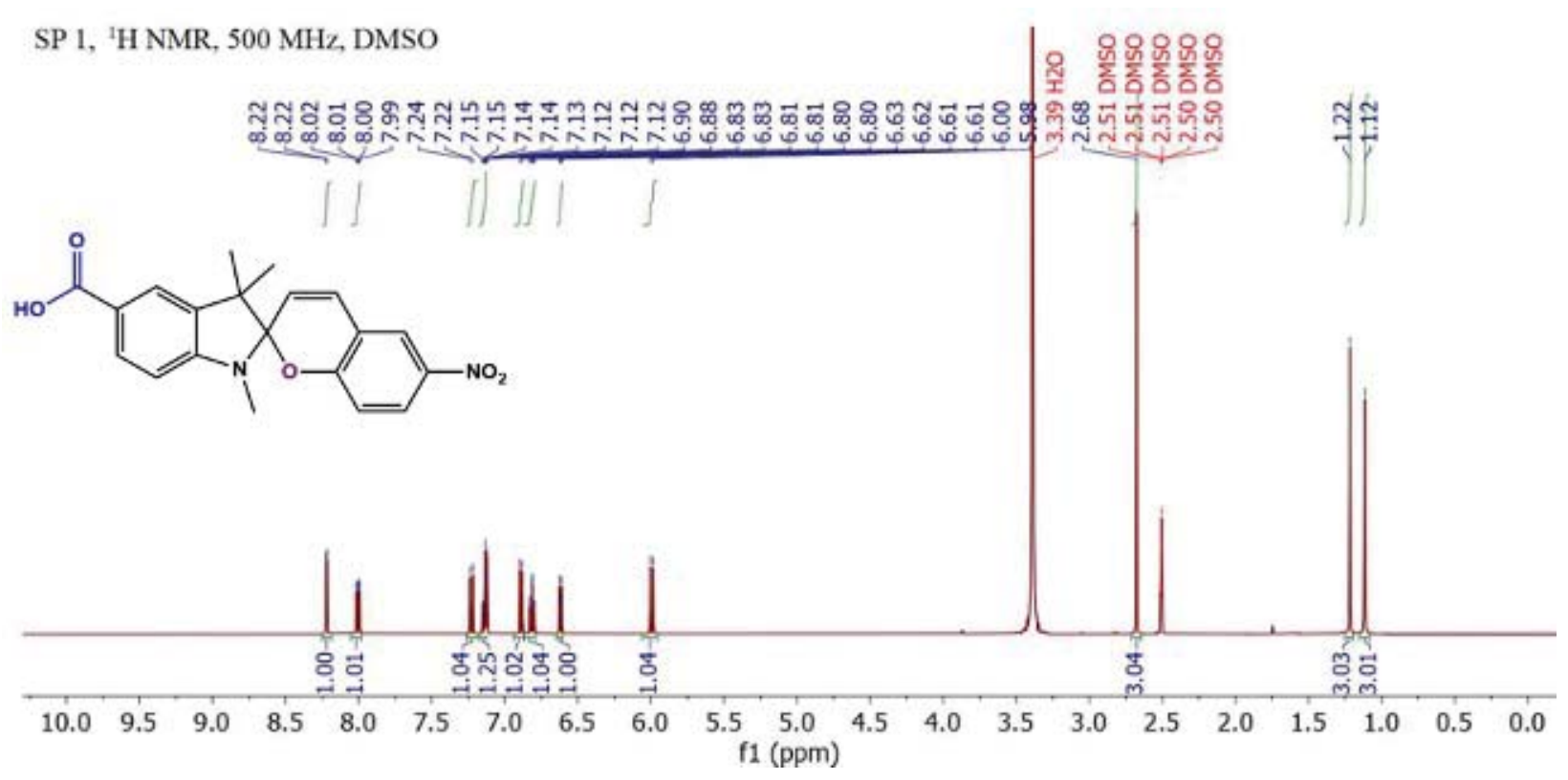

${ }^{13} \mathrm{C}\{1 \mathrm{H}\}$ for proton-decoupled carbon data.

SP $1,{ }^{13} \mathrm{C}$ NMR, $126 \mathrm{MHz}$, DMSO

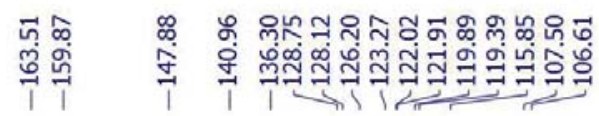
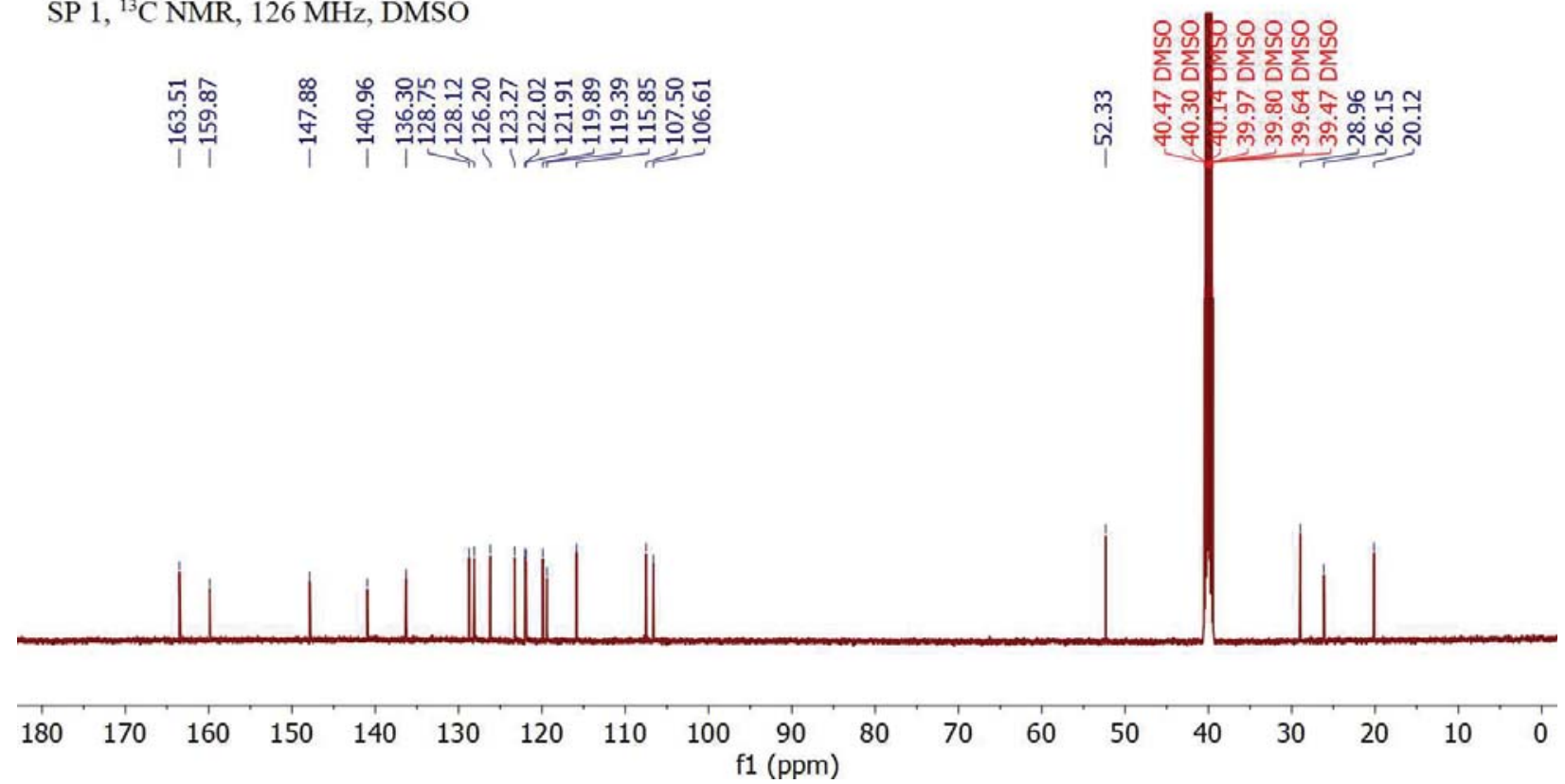
Single Mass Analysis

Tolerance $=5.0$ PPM I DBE: $\min =-1.5, \max =100.0$

Element prediction: Off

Number of isotope peaks used for i-FIT $=3$

Monoisotopic Mass, Even Electron lons

344 formula(e) evaluated with 1 results within limits (up to 50 closest results for each mass)

Elements Used:

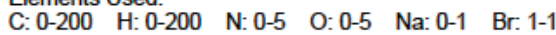

SP 2
Qtof_8 63 (2.370) AM (Cen,5, 80.00, Ar, 14000.0,558.36,0.70,LS 5); Sm (SG, 2x5.00); Cm (55:63)

1: TOF MS ES+

100 3 $344.1265 \quad 1.53 \mathrm{e}+003$

100
$\%$

Minimum:

Maximum:

5.0

$\begin{array}{ll} & -1.5 \\ 5.0 & 100.0\end{array}$

Mass

Calc. Mass $\mathrm{mDa}$

PPM

DBE

i-FIT

Formula

$344.1265 \quad 344.1263$

$\begin{array}{ll}0.3 & 0.8\end{array}$

10.5

14.5

C20 H19 $\mathrm{N}$ O3 $\mathrm{Na}$ 
SP 2, 'H NMR, $500 \mathrm{MHz}$, DMSO

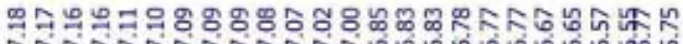

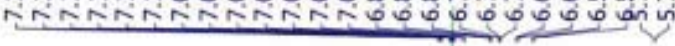<smiles>CN1c2ccc(C(=O)O)cc2C(C)(C)C12C=Cc1ccccc1O2</smiles>
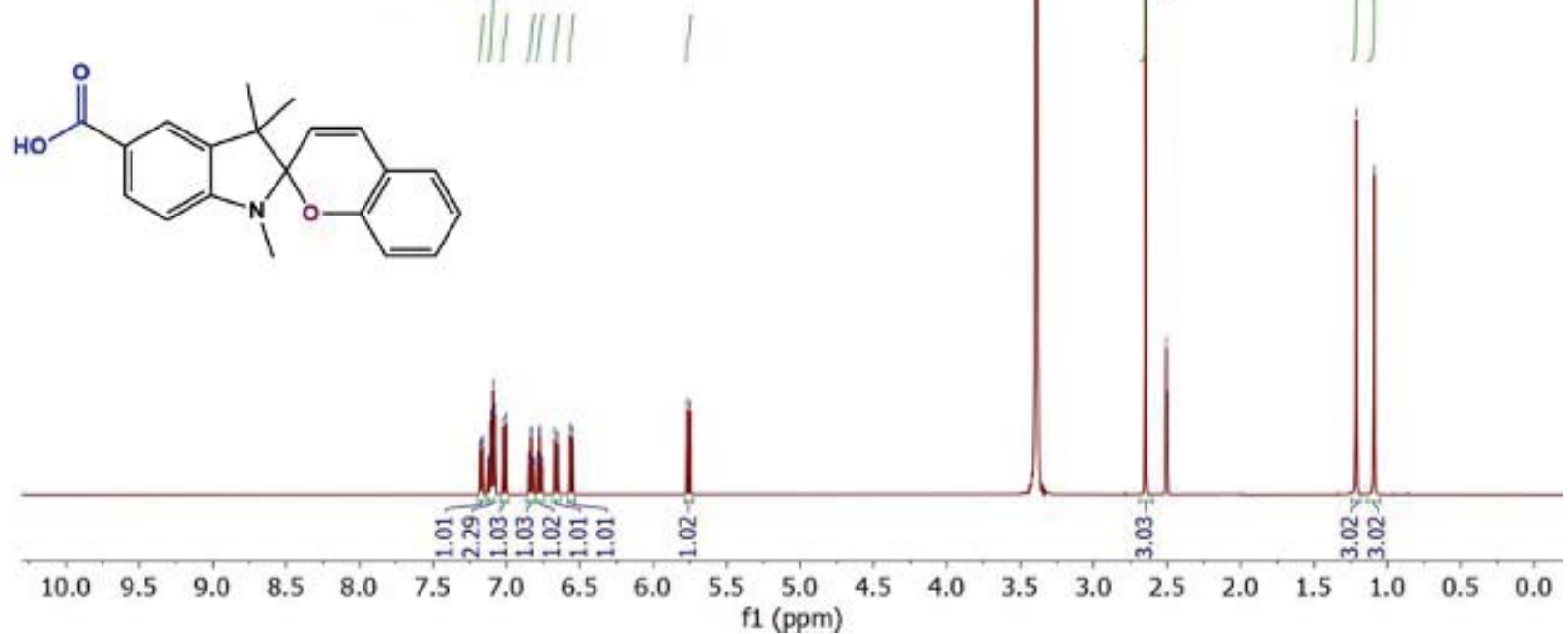

${ }^{13} \mathrm{C}\{1 \mathrm{H}\}$ for proton-decoupled carbon data.

SP 2, ${ }^{13} \mathrm{C}$ NMR, $126 \mathrm{MHz}$, DMSO

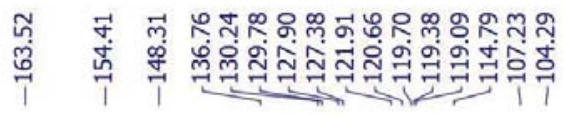
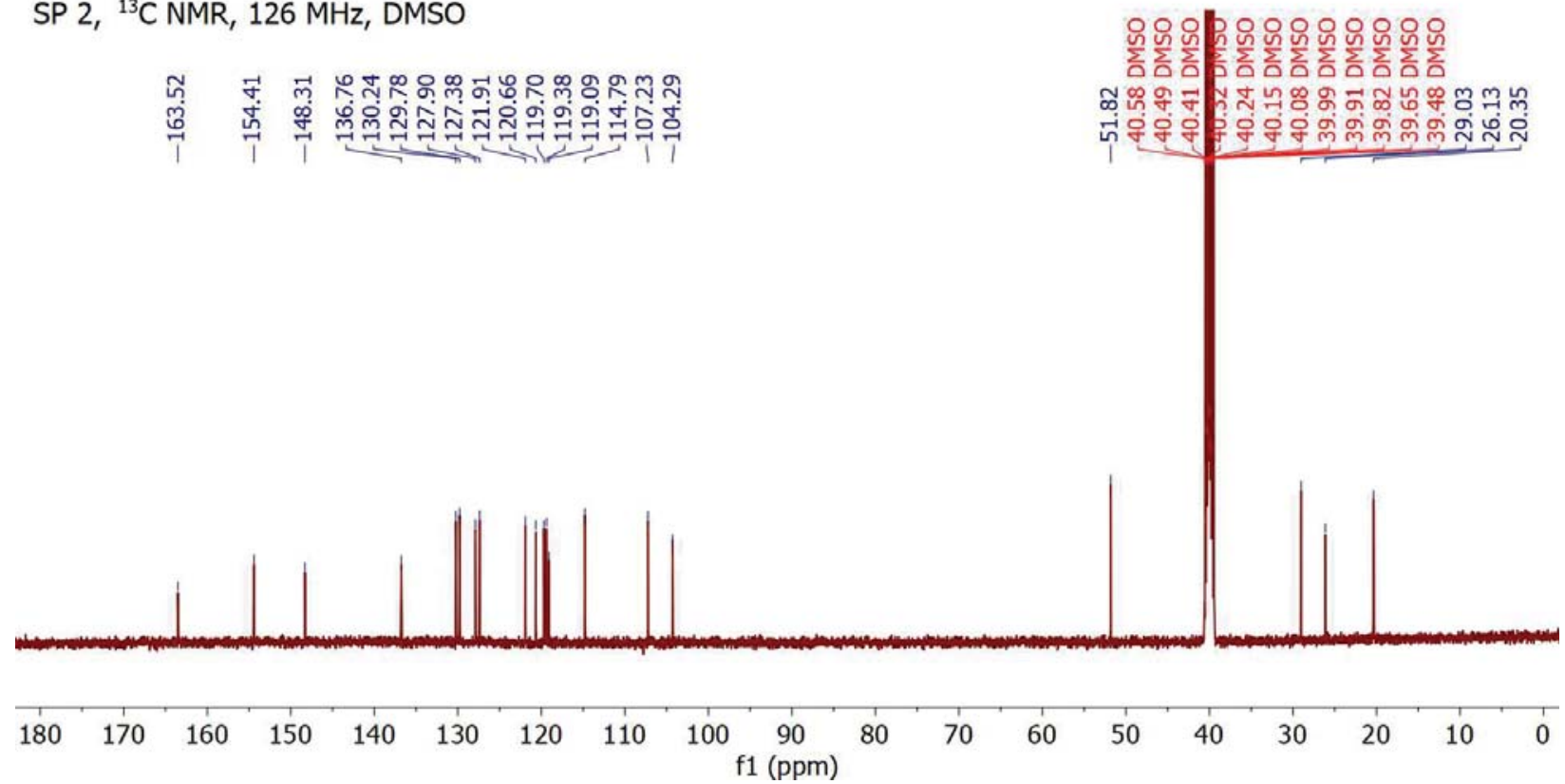
Single Mass Analysis

Tolerance $=5.0$ PPM I DBE: $\min =-1.5, \max =100.0$

Element prediction: Off

Number of isotope peaks used for i-FIT $=3$

Monoisotopic Mass, Even Electron lons

358 formula(e) evaluated with 1 results within limits (up to 50 closest results for each mass)

Elements Used:

$\begin{array}{lllll}\text { C: } 0-200 & \mathrm{H}: 0-200 & \mathrm{~N}: 0-5 & \mathrm{O}: 0-6 & \mathrm{Na}: 0-1\end{array}$

SP 3 Qtof_9 63 (2.370) AM (Cen,5, 80.00, Ar, 14000.0,558.36,0.70,LS 5); Sm (SG, 2x5.00); Cm (62:64)

1: TOF MS ES+

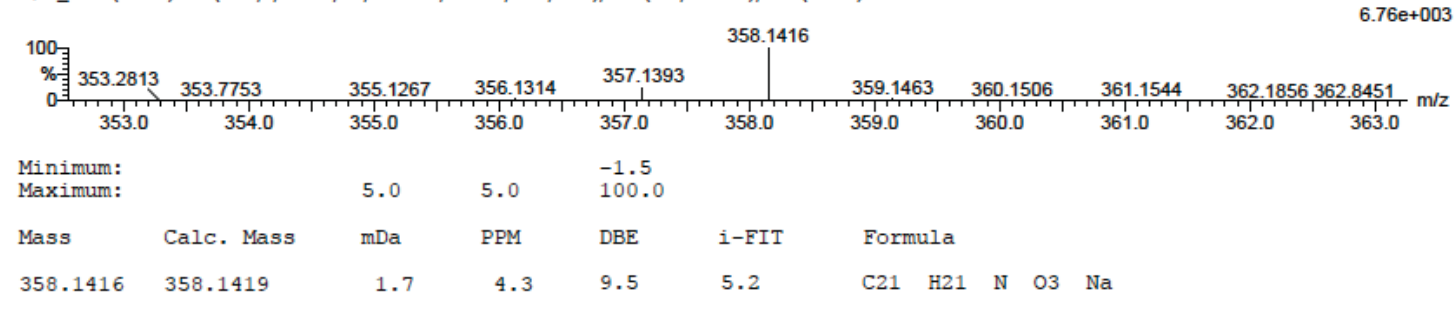




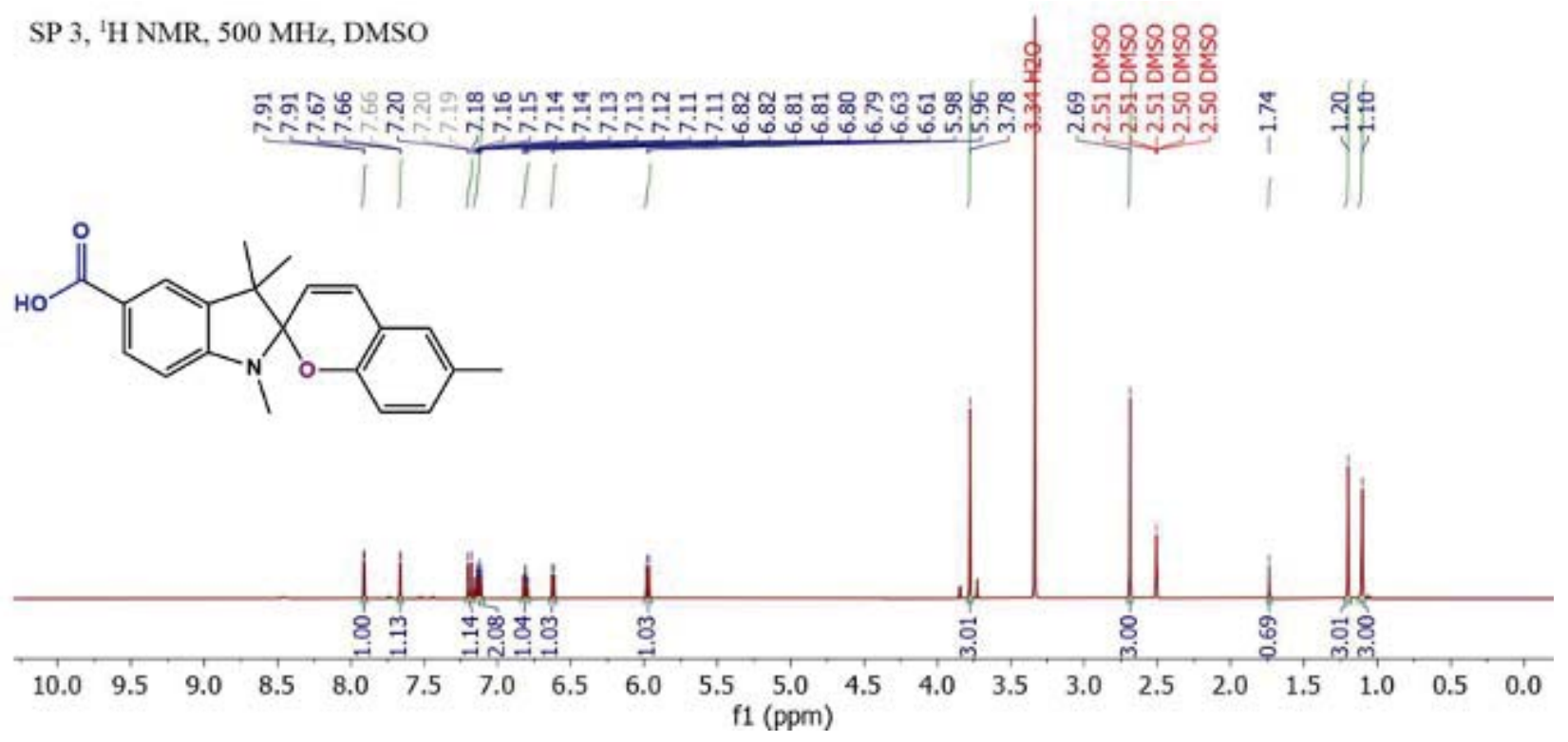

${ }^{13} \mathrm{C}\{1 \mathrm{H}\}$ for proton-decoupled carbon data.

SP $3,{ }^{13} \mathrm{C}$ NMR, $126 \mathrm{MHz}$, DMSO

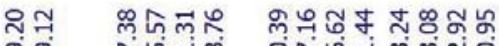

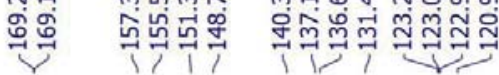

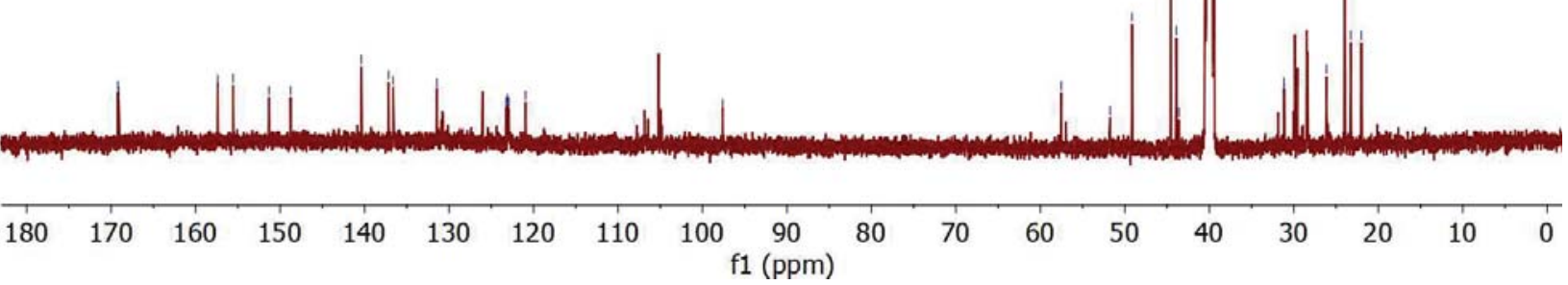


Single Mass Analysis

Tolerance $=5.0$ PPM I DBE: $\min =-1.5, \max =100.0$

Element prediction: Off

Number of isotope peaks used for i-FIT $=3$

Monoisotopic Mass, Even Electron lons

359 formula(e) evaluated with 1 results within limits (up to 50 closest results for each mass)

Elements Used

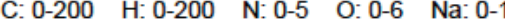

SP 4 Qtof_10 60 (2.268) AM (Cen,5, 80.00, Ar, 14000.0,558.36,0.70,LS 5); Sm (SG, 2x5.00); Cm (55:60)

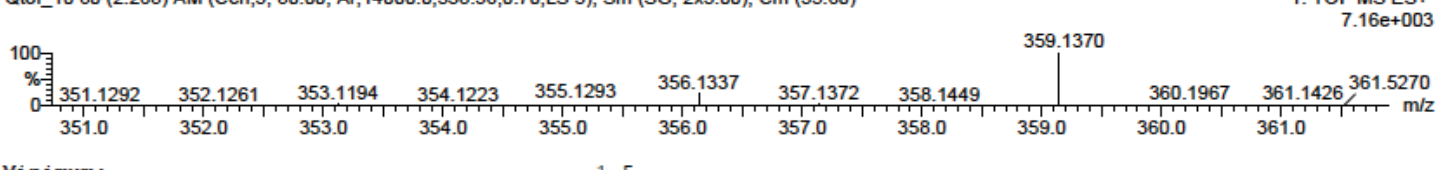

Minimum:

Maximum:

5.0

5.0

$-1.5$

Mass

Calc. Mass

$\mathrm{mDa}$

PPM

$\mathrm{DBE}$

i-FIT

Formula

$359.1370 \quad 359.1372$

$-0.1$

$-0.3$

12.5

11.5

$\mathrm{C} 20 \mathrm{H} 2 \mathrm{O} \quad \mathrm{N} 2 \quad \mathrm{O} 3 \quad \mathrm{Na}$ 
SP 4, ${ }^{1} \mathrm{H}$ NMR, $500 \mathrm{MHz}$, DMSO

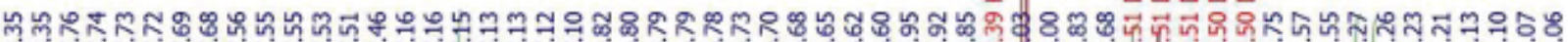

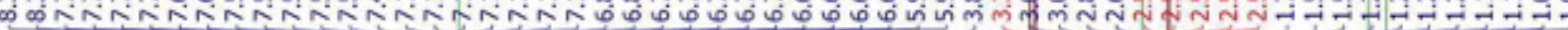<smiles>CC=CC(C)C</smiles>

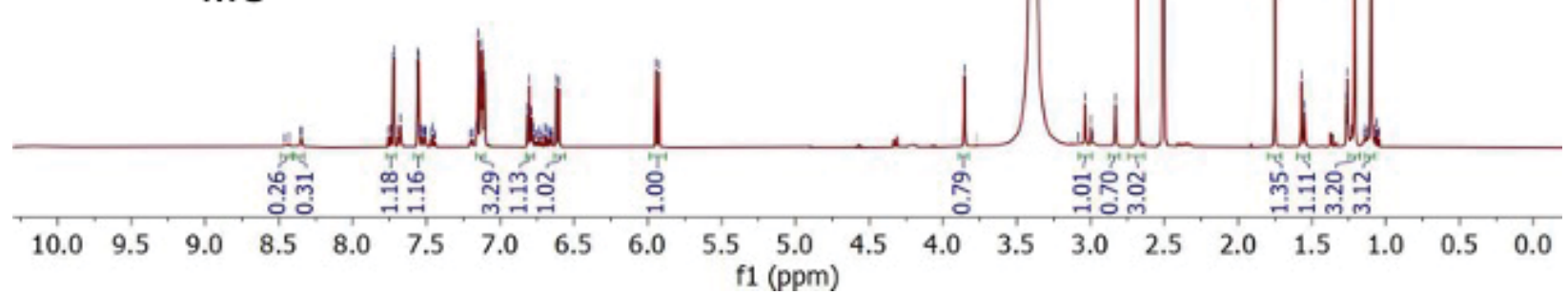

${ }^{13} \mathrm{C}\{1 \mathrm{H}\}$ for proton-decoupled carbon data.

$\mathrm{SP} 4,{ }^{13} \mathrm{C}$ NMR, $126 \mathrm{MHz}$, DMSO

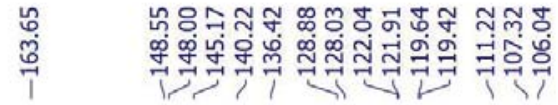

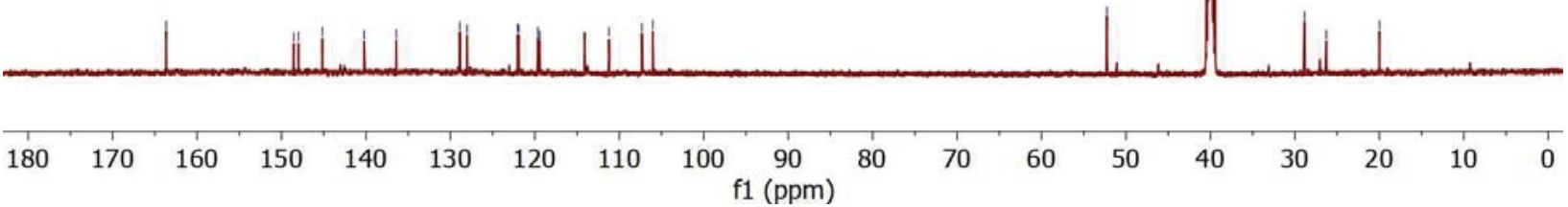


Single Mass Analysis

Tolerance $=5.0$ PPM I DBE: $\min =-1.5, \max =100.0$

Element prediction: Off

Number of isotope peaks used for i-FIT $=3$

Monoisotopic Mass, Even Electron lons

360 formula(e) evaluated with 1 results within limits (up to 50 closest results for each mass)

Elements Used:

$\begin{array}{lllll}\text { C: } 0-200 & \mathrm{H}: 0-200 & \mathrm{~N}: 0-5 & \mathrm{O}: 0-6 & \mathrm{Na}: 0-1\end{array}$

Qtof_11 63 (2.370) AM (Cen,5, 80.00, Ar, 14000.0,558.36,0.70,LS 5); Sm (SG, 2x5.00); Cm (58:63)

1: TOF MS ES+

$2.11 \mathrm{e}+003$

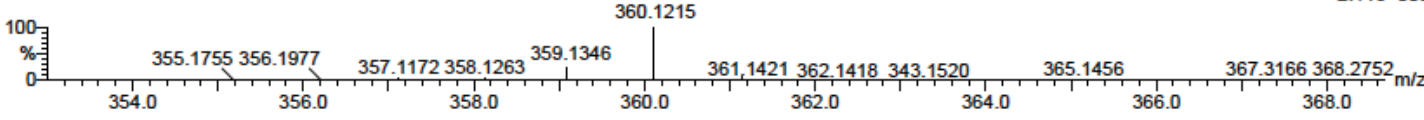

Minimum:

Maximum:

$-1.5$

Mass Calc. Mass mDa PPM DBE

$360.1215 \quad 360.1211$

$-1.5 \quad-4.4$

12.5

i-FIT

Formula 


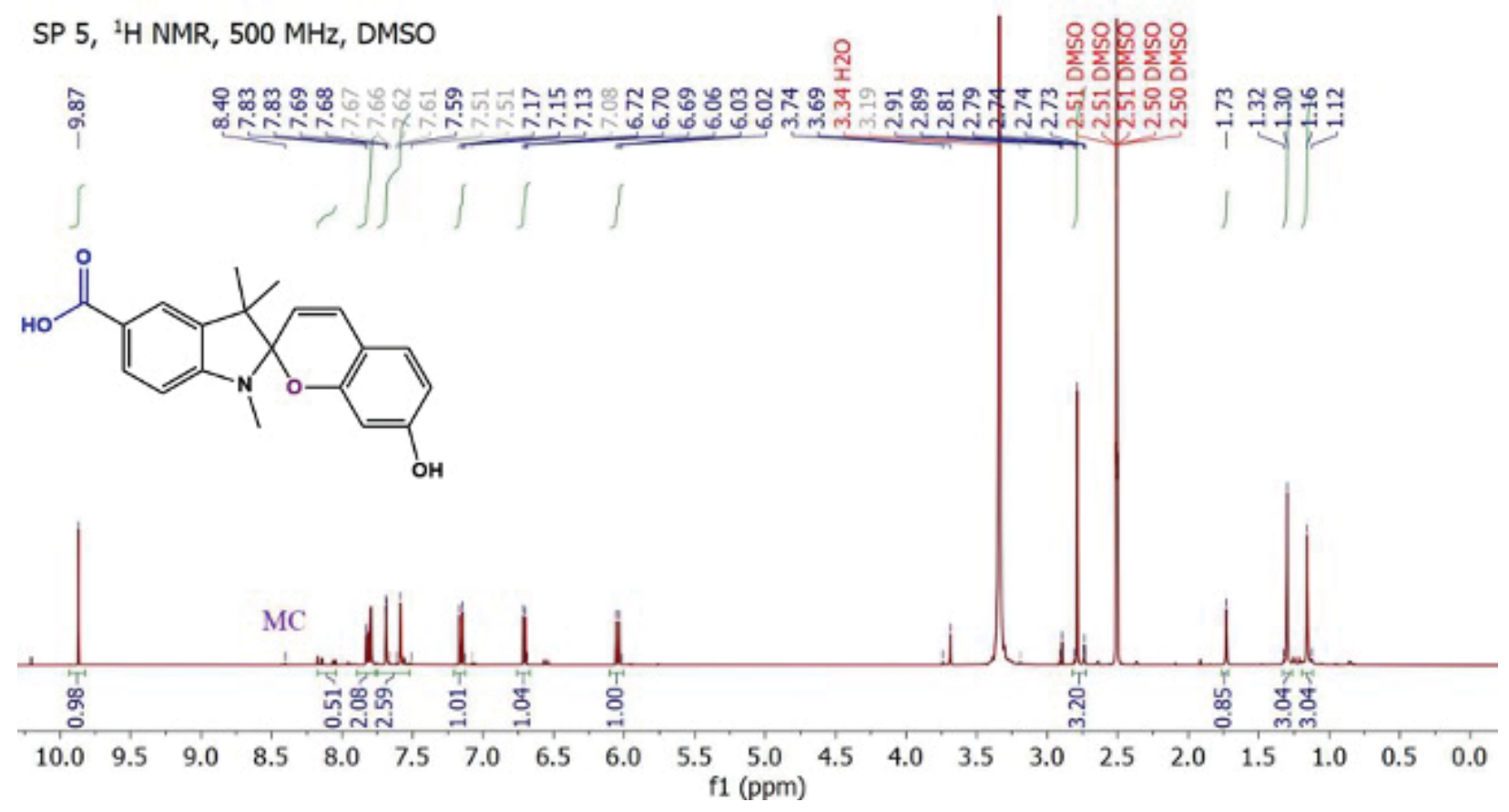

${ }^{13} \mathrm{C}\{1 \mathrm{H}\}$ for proton-decoupled carbon data.

SP $5,{ }^{13} \mathrm{C}$ NMR, $126 \mathrm{MHz}$, DMSO
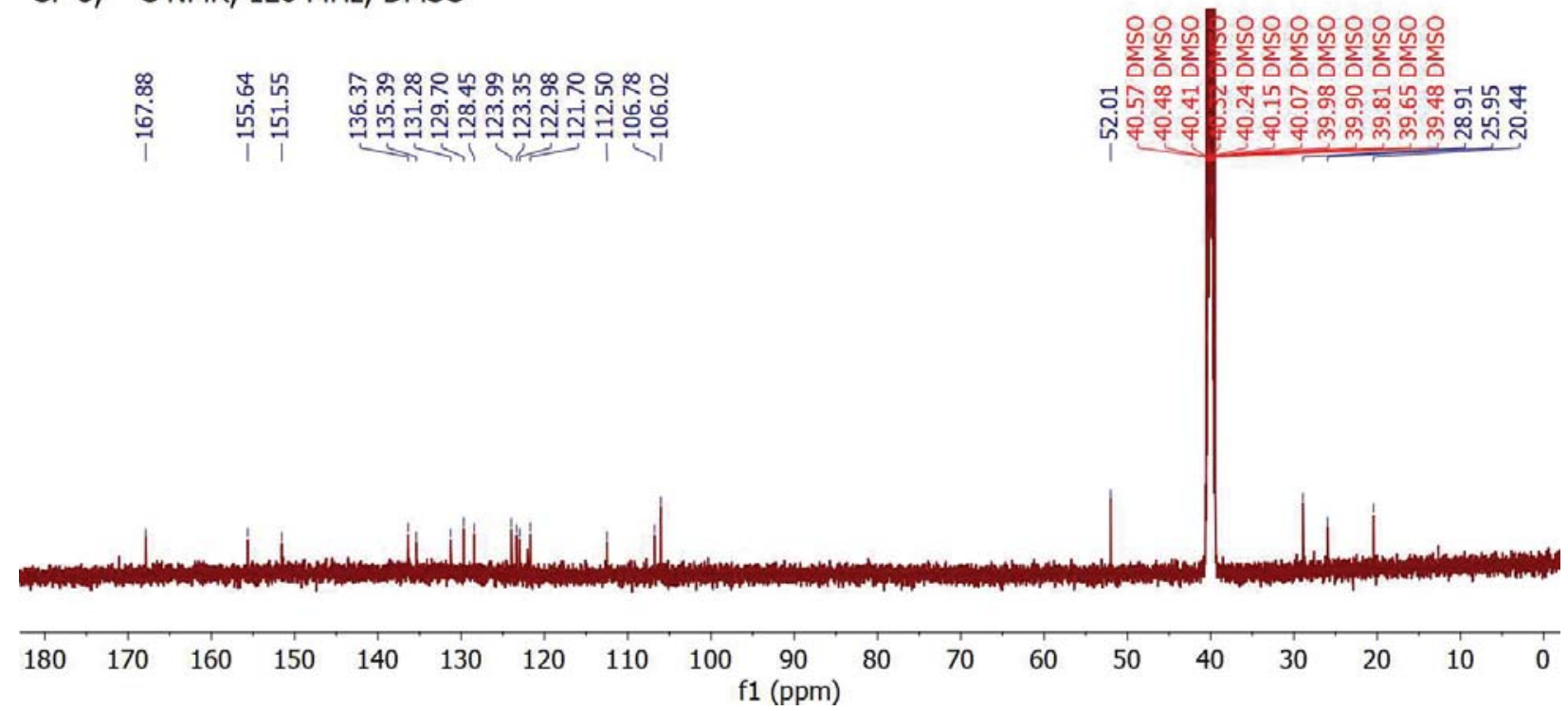
Single Mass Analysis

Tolerance $=5.0$ PPM $/$ DBE: $\min =-1.5, \max =100.0$

Element prediction: Off

Number of isotope peaks used for i-FIT $=3$

Monoisotopic Mass, Even Electron lons

438 formula(e) evaluated with 1 results within limits (up to 50 closest results for each mass)

Elements Used:

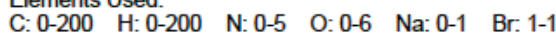

SP 6 Qtof_12 60 (2.268) AM (Cen,5, 80.00, Ar, 14000.0,558.36,0.70,LS 5); Sm (SG, 2x5.00); Cm (56:61)

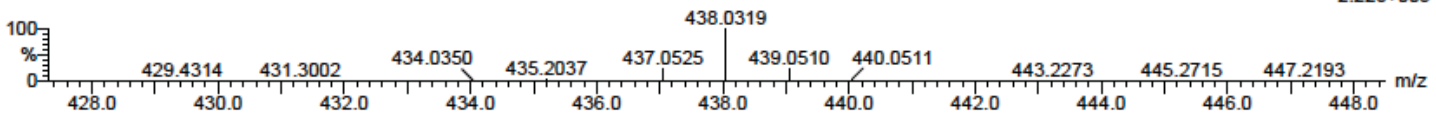

Minimum:

Maximum:

$\begin{array}{lll}5.0 & 5.0 & -1.5 \\ & & 100.0\end{array}$

Mass

Calc. Mass

$\mathrm{mDa}$

PPM

$\mathrm{DBE}$

i-FIT

Formula

438.0319

438.0317

1.5

3.6

8.5

4.2

$\begin{array}{llllll}\mathrm{C} 20 & \mathrm{H} 18 & \mathrm{~N} & \mathrm{O} 4 & \mathrm{Na} & \mathrm{Br}\end{array}$ 
SP 6, ${ }^{1} \mathrm{H}$ NMR, $500 \mathrm{MHz}$, DMSO

กิ<smiles>CN1c2ccc(C(=O)O)cc2C(C)(C)C12C=Cc1c(Br)cc(O)cc1O2</smiles>

IS IS S S
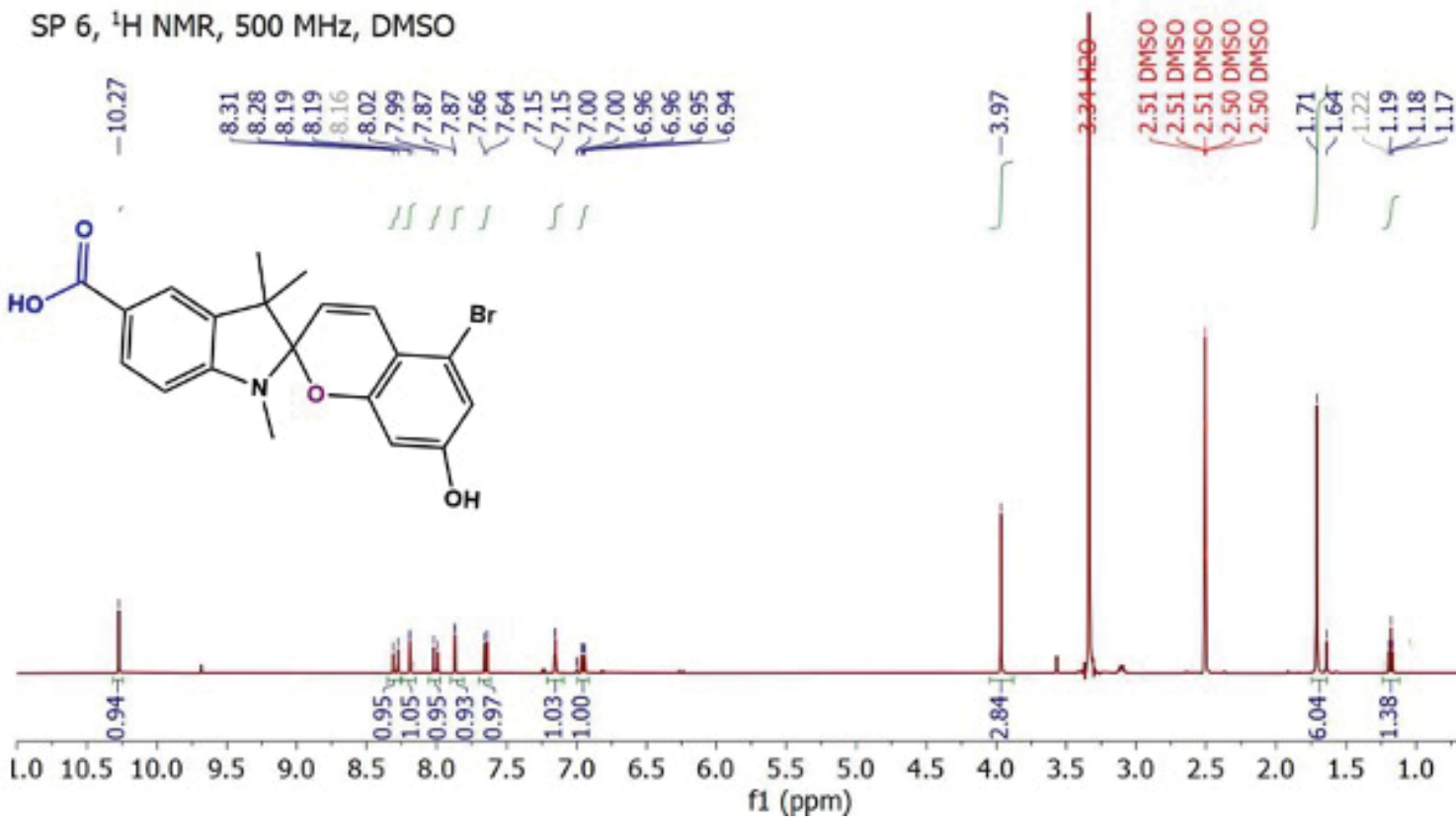

${ }^{13} \mathrm{C}\{1 \mathrm{H}\}$ for proton-decoupled carbon data.

SP $6,{ }^{13} \mathrm{C}$ NMR, $126 \mathrm{MHz}$, DMSO

ํㅠ ए ए

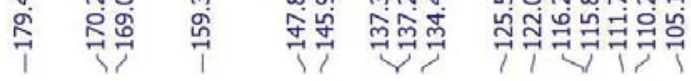
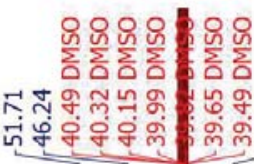

누워ำ
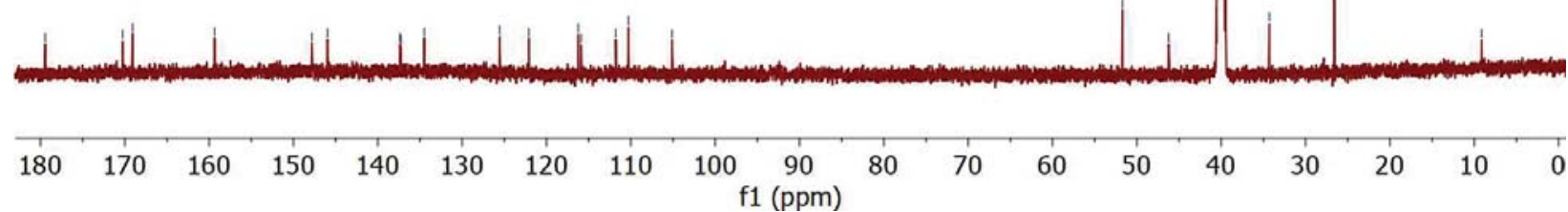
Single Mass Analysis

Tolerance $=5.0$ PPM I DBE: $\min =-1.5, \max =100.0$

Element prediction: Off

Number of isotope peaks used for i-FIT $=3$

Monoisotopic Mass, Even Electron lons

382 formula(e) evaluated with 1 results within limits (up to 50 closest results for each mass)

Elements Used:

$\begin{array}{lllll}\text { C: } 0-200 & \text { H: } 0-200 & \text { N: } 0-5 & \text { O: } 0-6 & \text { Na: } 0-1\end{array}$

Qtof_002 68 (2.572) AM (Cen,5, 80.00, Ar, 14000.0,558.36,0.70,LS 5); Sm (SG, 2×5.00); Cm (68:79)

1: TOF MS ES+

382.1050

$3.16 \mathrm{e}+002$

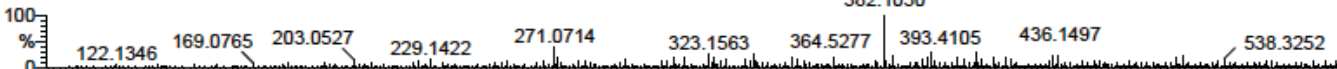

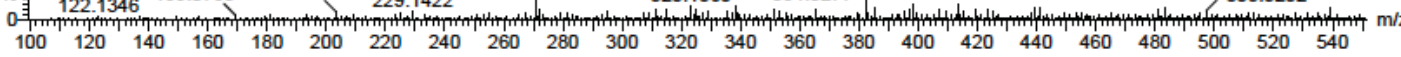

Minimum:

Maximum:

$-1.5$

Mass Calc. Mass mDa PPM DBE

$382.1050 \quad 382.1055$

$\begin{array}{ll}-0.5 & -1.8\end{array}$

10.5

i-FIT

Formula

23.8

C22 H17 N2 O2 Na 
la, ${ }^{1} \mathrm{H}$ NMR, $500 \mathrm{MHz}$, DMSO

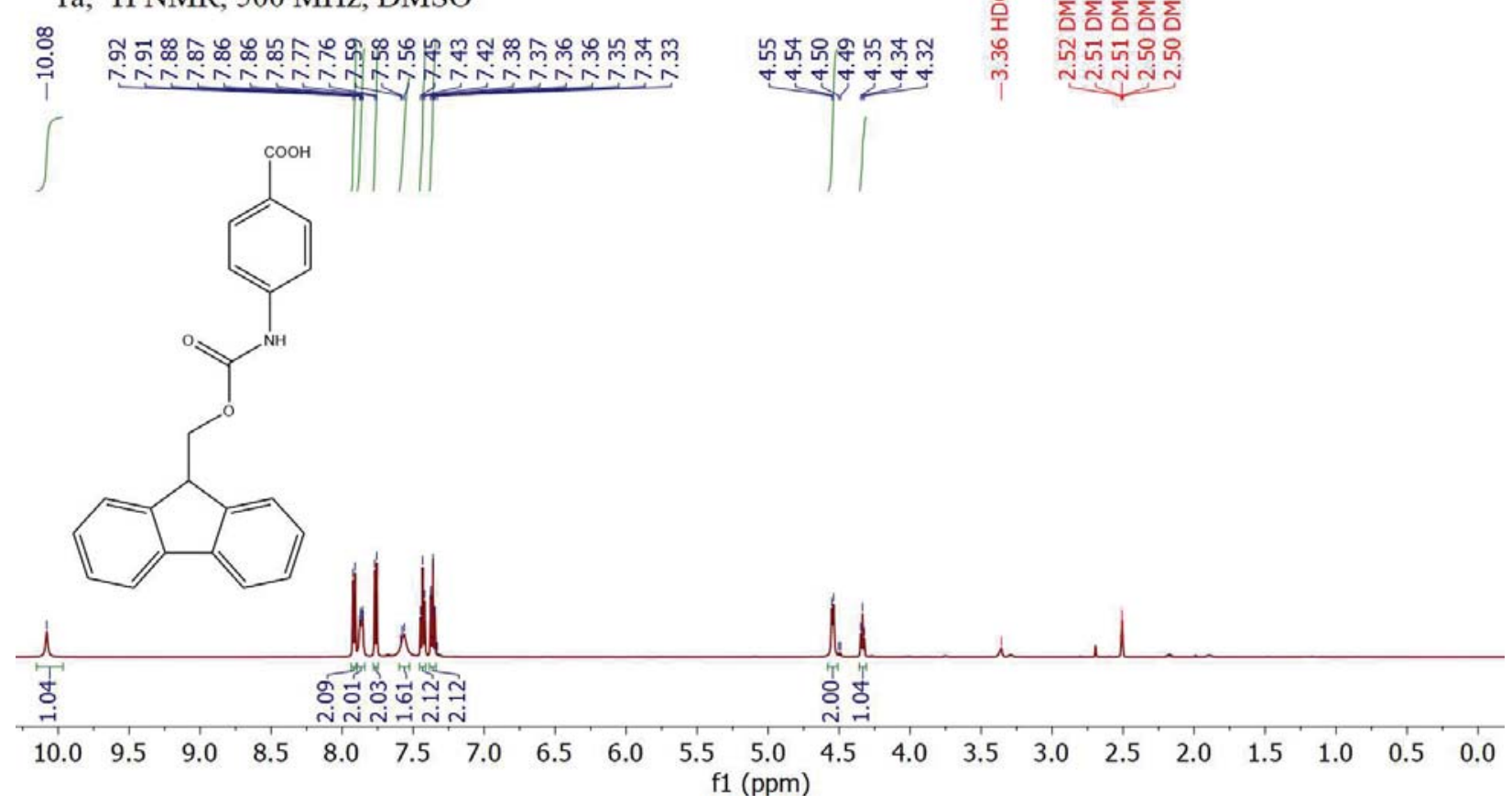

${ }^{13} \mathrm{C}\{1 \mathrm{H}\}$ for proton-decoupled carbon data.

1a, ${ }^{13} \mathrm{C}$ NMR, $126 \mathrm{MHz}$, DMSO

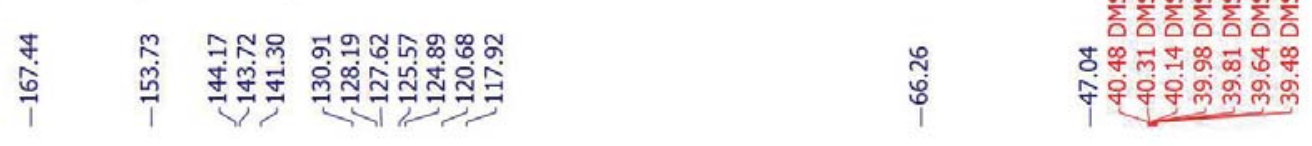

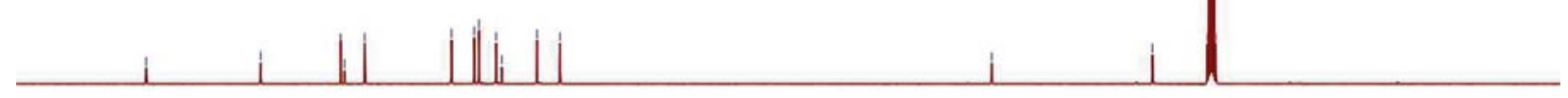

180

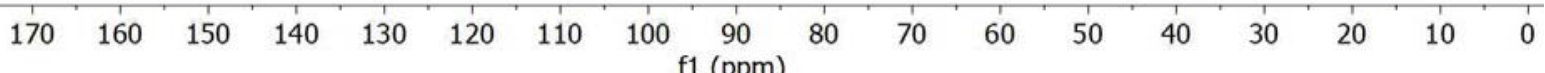


Single Mass Analysis

Tolerance $=5.0$ PPM I DBE: $\min =-1.5, \max =100.0$

Element prediction: Off

Number of isotope peaks used for i-FIT = 3

Monoisotopic Mass, Even Electron lons

231 formula(e) evaluated with 1 results within limits (up to 50 closest results for each mass)

Elements Used:

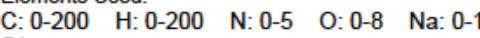

Q1tof_01 60 (2.336) AM (Cen,5, 80.00, Ar, 14000.0,558.36,0.70,LS 5); Sm (SG, 2x5.00); Cm (59:62)

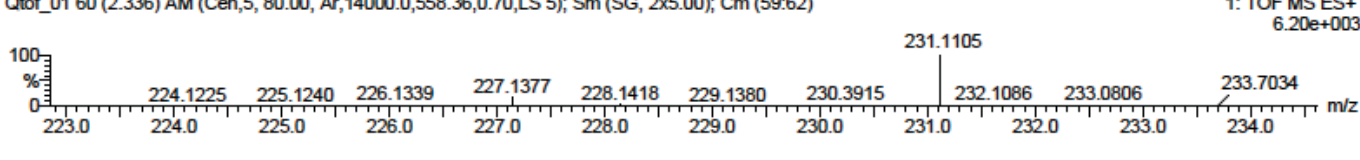

Minimum:

Maximum:

$\begin{array}{lll}5.0 & 5.0 & -1.5 \\ & 100.0\end{array}$

Mass

Calc. Mass

mDa

PPM

100.0

231.1105

231.1109

$-0.5$

$-2.2$

8.5

i-FIT

Formula 
B1, ${ }^{1} \mathrm{H}$ NMR, $500 \mathrm{MHz}$, DMSO
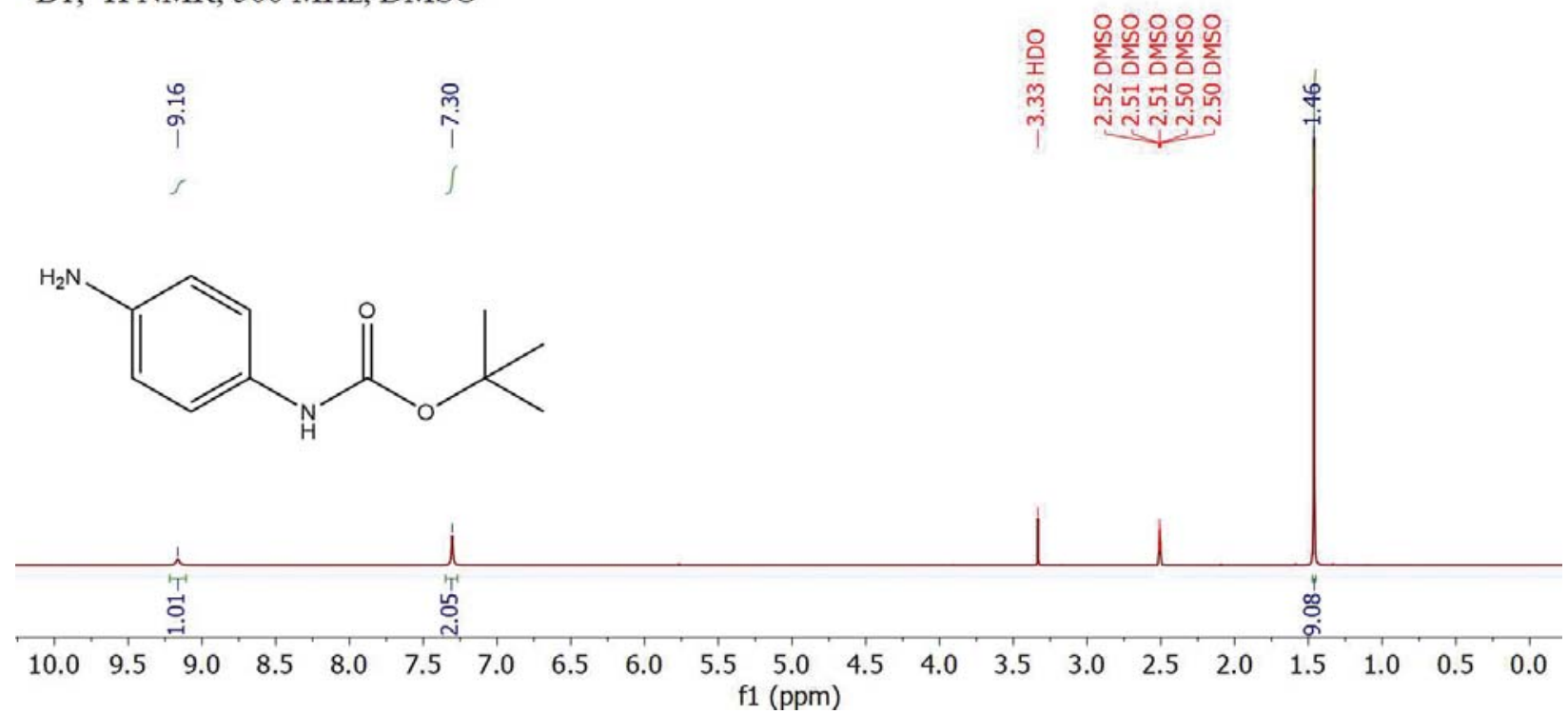

${ }^{13} \mathrm{C}\{1 \mathrm{H}\}$ for proton-decoupled carbon data.

$\mathrm{B} 1,{ }^{13} \mathrm{C}$, NMR, $126 \mathrm{MHz}$, DMSO

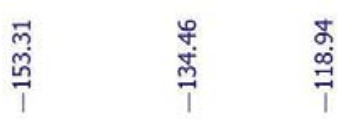

$\stackrel{\infty}{\stackrel{\infty}{\rightarrow}}$
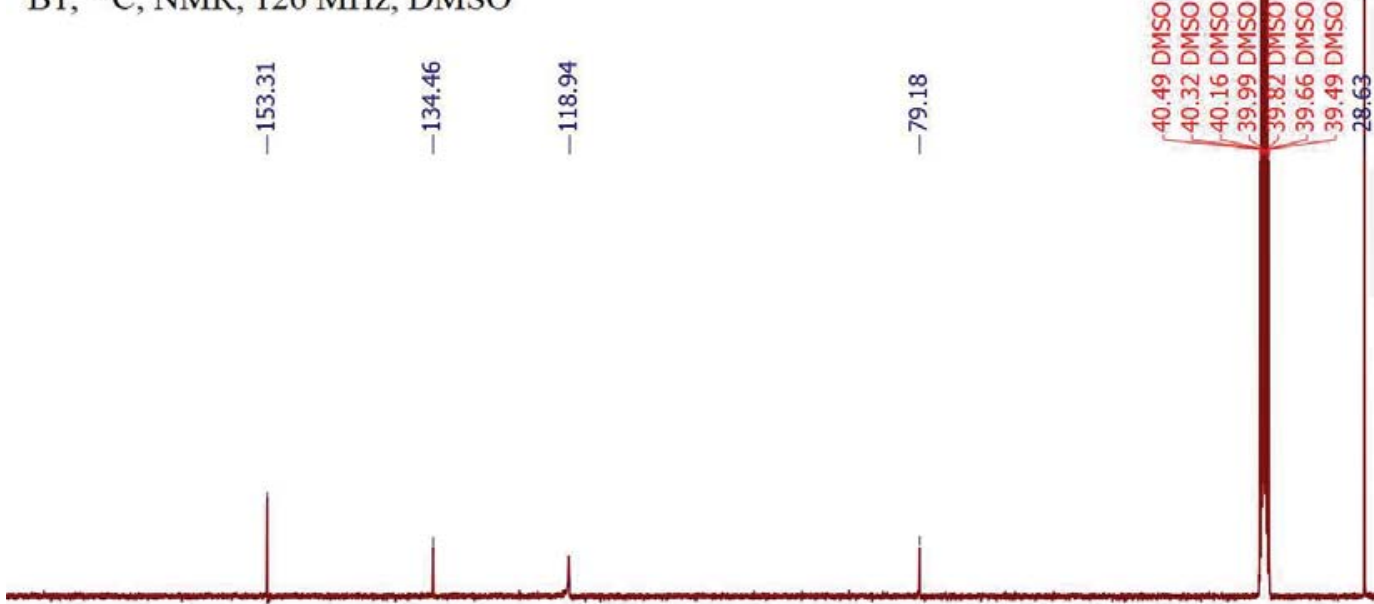

$\begin{array}{llllllllllllllllll}180 & 170 & 160 & 150 & 140 & 130 & 120 & 110 & 100 \begin{array}{c}90 \\ \mathrm{f} 1(\mathrm{ppm})\end{array} & 80 & 70 & 60 & 50 & 40 & 30 & 20 & 10 & 0\end{array}$


Inj Volume : $5.000 \mu \mathrm{l}$

1. Method .... : Symmetry C-16 75*4.6mm,3.5mm

Mobile phase: A) $0.1 \mathrm{HCOOH}$ in $\mathrm{H} 2 \mathrm{O}$, B) $\mathrm{ACN}$

FL $\backslash$ low $1.0 \mathrm{ml} / \mathrm{min}$, Diluent: $\mathrm{ACN}: \mathrm{H} 2 \mathrm{O}$ (90:10)

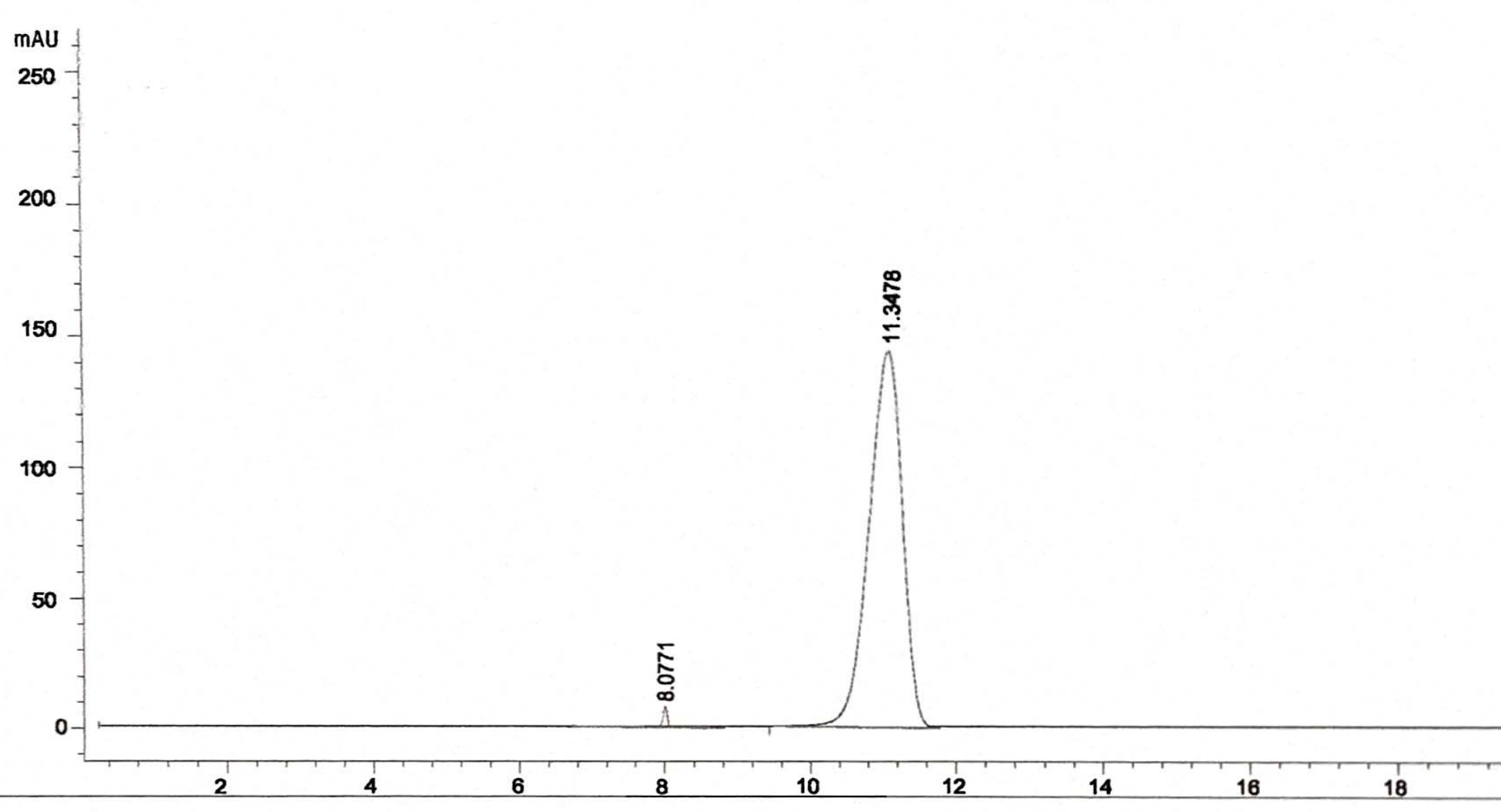

Area Percent Report

ignal 1: VWD1 A, Wavelength=273 nm

ak RetTime Type Area Area

[min] [mAU*s] \%

$-1-----11--------11-------1$

$\begin{array}{llll}1 & 8.0771 & 113.6272 & 3.8105\end{array}$

$\begin{array}{llll}2 & 11.3478 & 2868.2546 & 96.1894\end{array}$ 
FL \low $1.0 \mathrm{ml} / \mathrm{min}$, Diluent: $A C N: H 2 O$ (90:10)

เั่

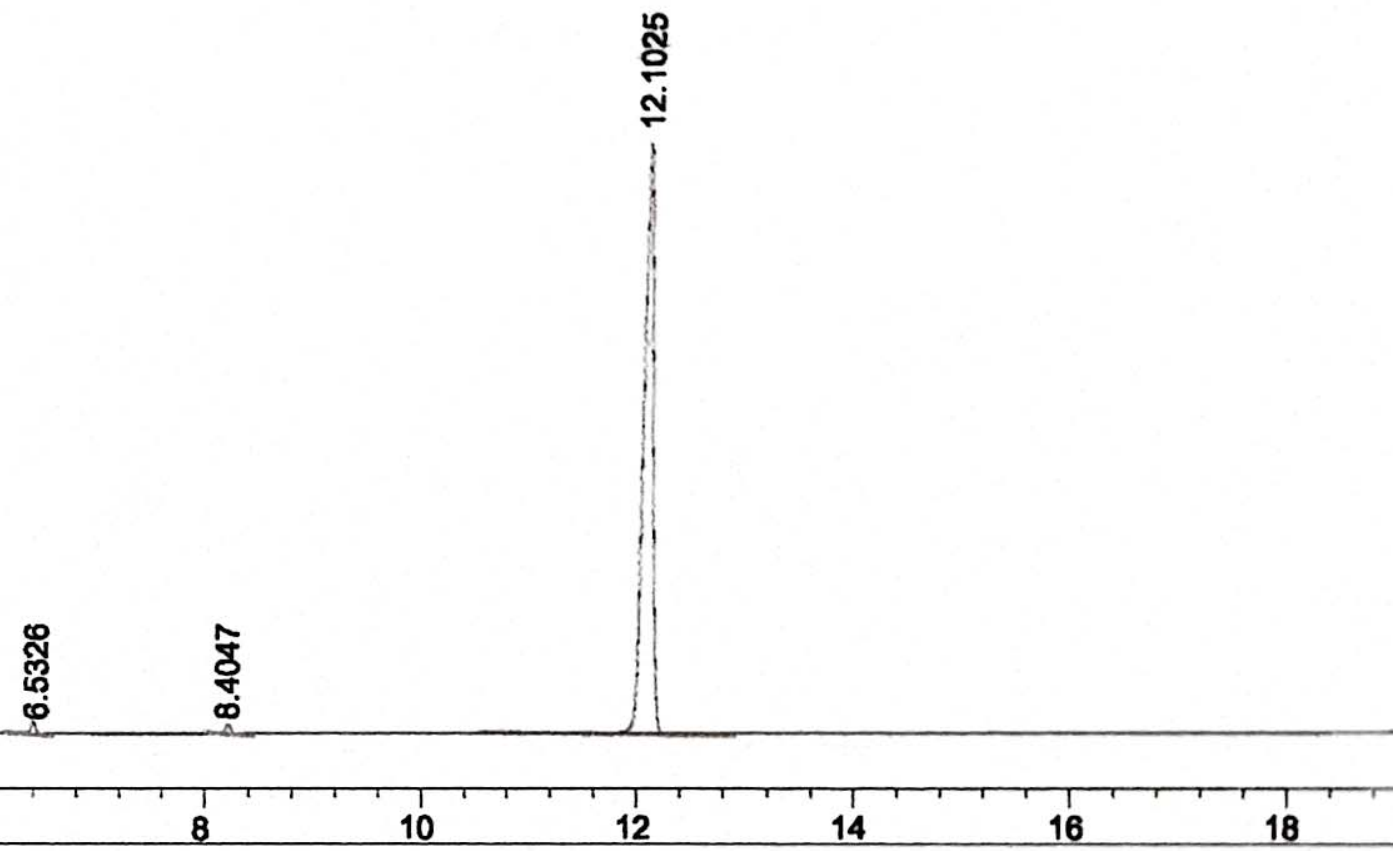

Area Percent Report

hal 1: VWD1 A, Wavelength $=273 \mathrm{~nm}$

\begin{tabular}{ccc}
$\begin{array}{c}\text { RetTime } \\
\text { [min] }\end{array}$ & $\begin{array}{c}\text { Area Area } \\
\text { [mAU*s] }\end{array}$ & $\begin{array}{c}\% \\
0\end{array}$ \\
\hline 6.5326 & 55.3649 & 1.7457 \\
8.4047 & 67.9486 & 2.1425 \\
12.1025 & 3048.0279 & 96.1118
\end{tabular}


Sample Name: AzO 3

Acq. Operator : SYSTEM
Sample Operator : SYSTEM

Inj : 1

Inj Volume : $5.000 \mathrm{\mu l}$

Acq. Method

: Symmetry C-16 75*4.6mm,3.5mm

Mobile phase: A) $0.1 \mathrm{HCOOH}$ in H2O, B) ACN

FL $\backslash$ low $1.0 \mathrm{ml} / \mathrm{min}$, Diluent: ACN:H2O (90:10)

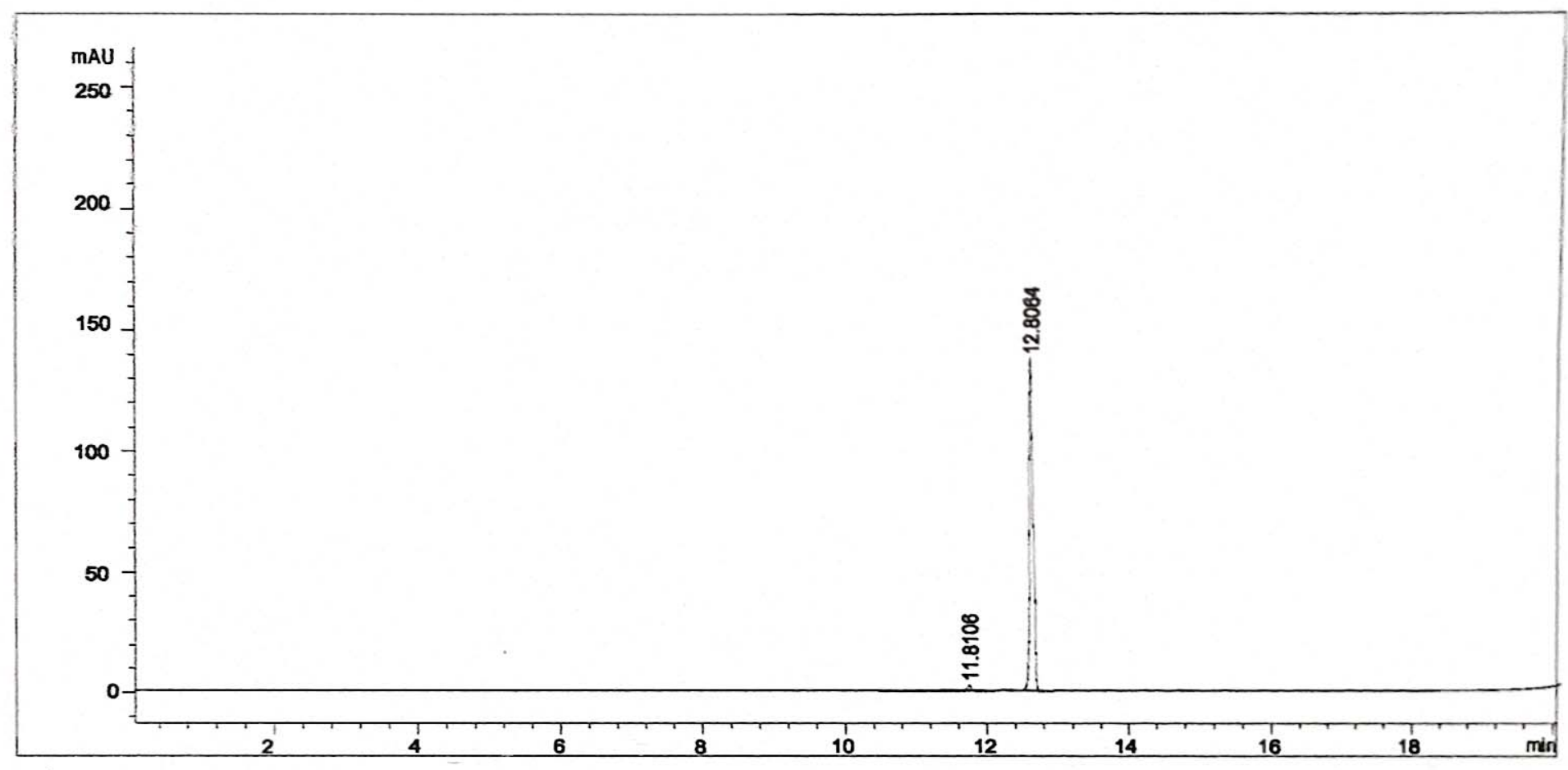

Area Percent Report

Signal 1: VWD1 A, Wavelength $=273 \mathrm{~nm}$

\begin{tabular}{cccc}
$\begin{array}{c}\text { Peak RetTime Type Area } \\
\text { [min] }\end{array}$ & $\begin{array}{c}\text { Area } \\
{\left[\mathrm{mAU} U^{*}\right]}\end{array}$ & $\begin{array}{c}\text { 8 } \\
\text { [min] }\end{array}$ \\
\hdashline 1 & 11.8106 & 44.0641 & 0.6716 \\
2 & 12.8064 & 6516.8346 & 99.3283
\end{tabular}


Sample Operator : SYSTEM

Inj : 1

Inj Volume : $5.000 \mathrm{\mu l}$

Acq. Method : Symmetry C-16 75*4.6mm,3.5mm

Mobile phase: A) $0.1 \mathrm{HCOOH}$ in $\mathrm{H} 2 \mathrm{O}$, B) $\mathrm{ACN}$

FI $\backslash$ low $1.0 \mathrm{ml} / \mathrm{min}$, Diluent: $\mathrm{ACN}: \mathrm{H} 2 \mathrm{O}$ (90:10)

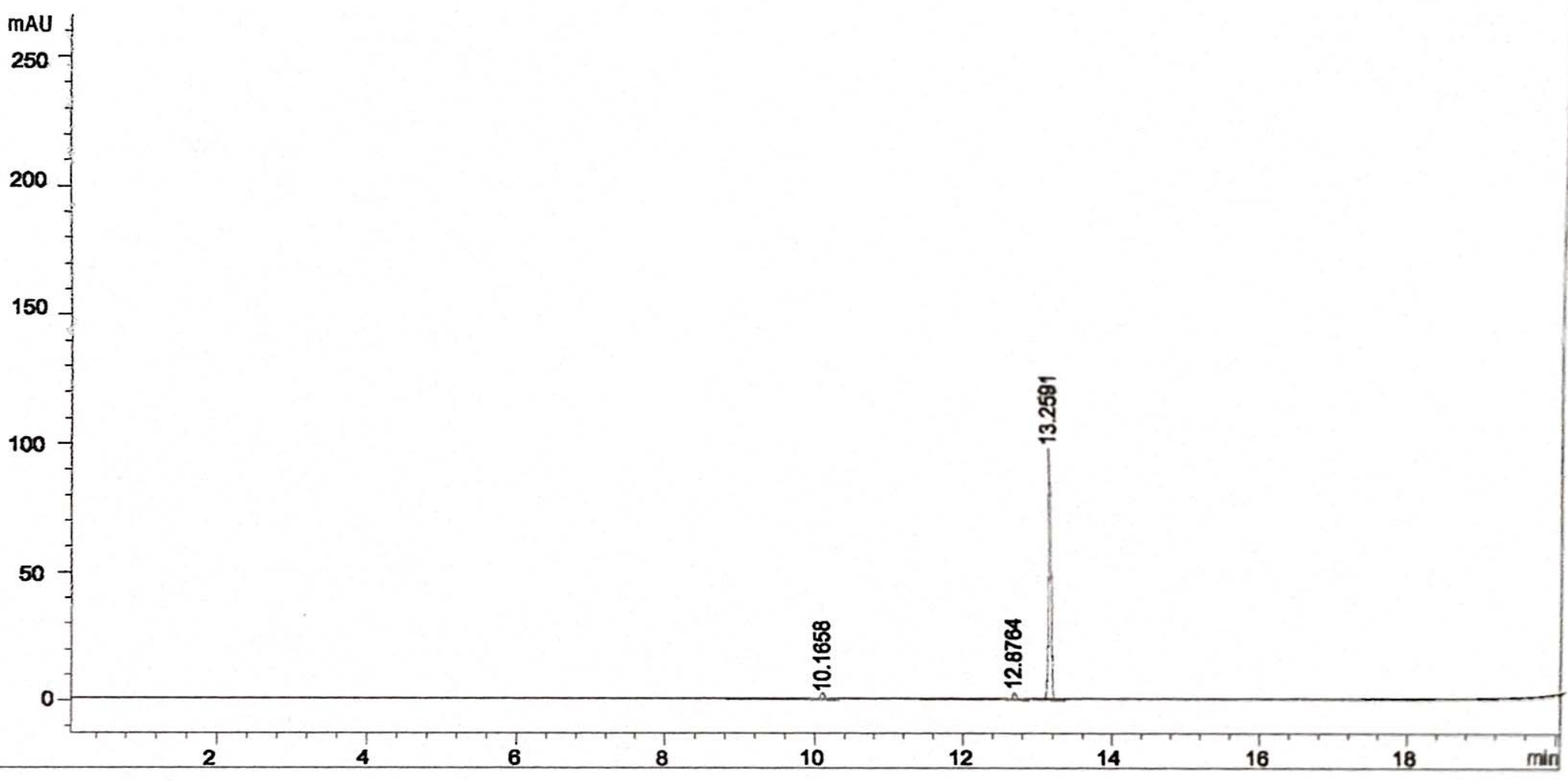

Area Percent Report

Signal 1: VWD1 A, Wavelength=273 nm

Peak RetTime Type Area

Area

[min] [mAU*s]

웅

$-1-----11--------11------1$

$\begin{array}{llll}1 & 10.1658 & 32.6579 & 1.0336\end{array}$

$2 \quad 12.8764 \quad 37.3516 \quad 1.1822$

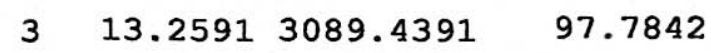




\section{Acq. Operator : SYSTEM \\ Sample Operator : SYSTEM}

Inj : 1

Inj Volume : $5.000 \mu \mathrm{l}$

Acq. Method

: Symmetry C-16 $75 * 4.6 \mathrm{~mm}, 3.5 \mathrm{~mm}$

Mobile phase: A) $0.1 \mathrm{HCOOH}$ in $\mathrm{H} 2 \mathrm{O}$, B) ACN

FL $\backslash$ low $1.0 \mathrm{ml} / \mathrm{min}$, Diluent: $\mathrm{ACN}: \mathrm{H} 2 \mathrm{O}$ (93:7)

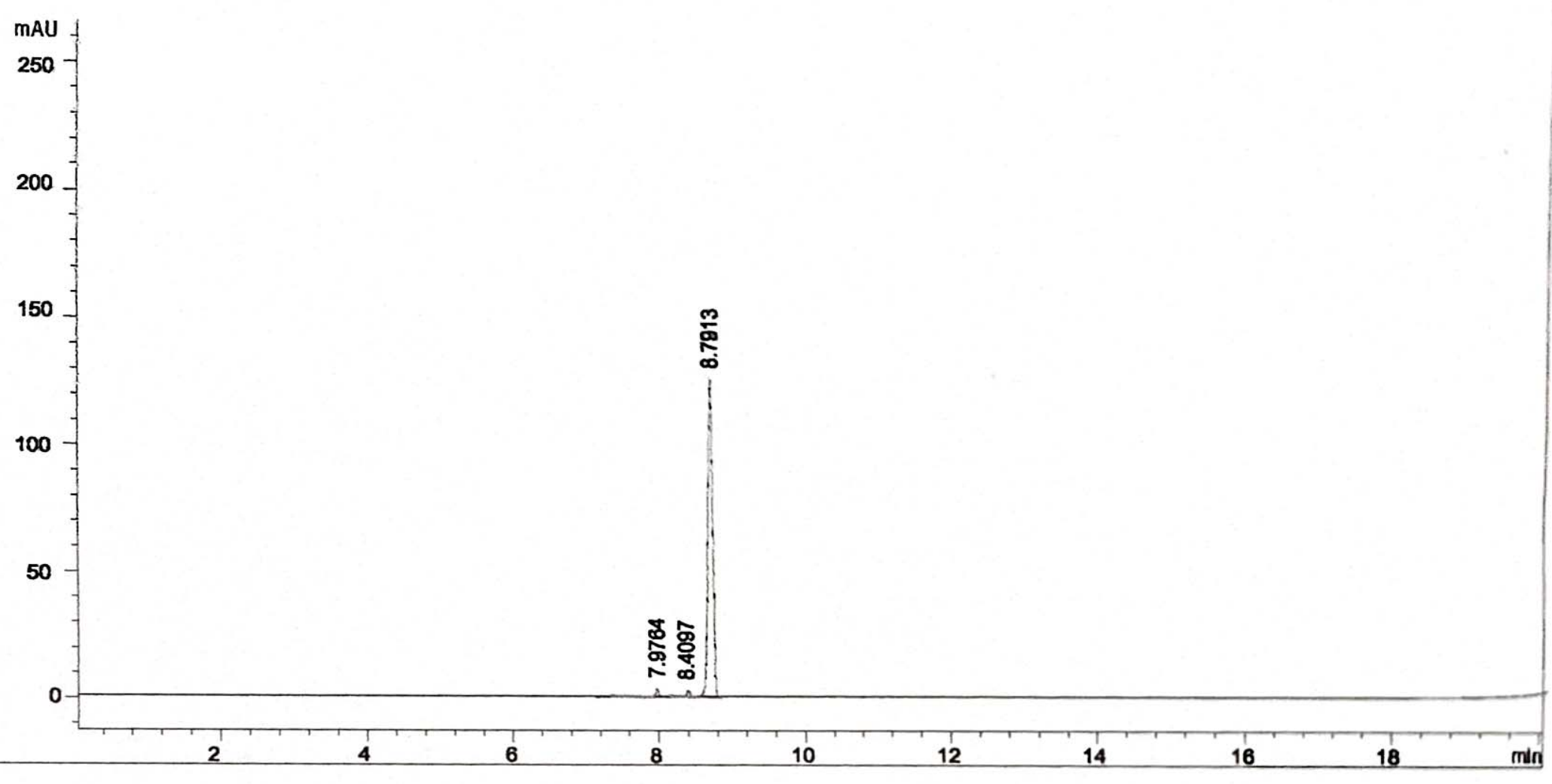

Area Percent Report

Signal 1: VWD1 A, Wavelength=273 nm

\begin{tabular}{cccc} 
Peak RetTime Type Area & Area \\
\# & [min] & [mAU*s] & \multicolumn{1}{c}{$\begin{array}{c}8 \\
8\end{array}$} \\
\hline 1 & 7.9764 & 29.0679 & 0.6926 \\
2 & 8.4097 & 31.2879 & 0.7455 \\
3 & 8.7913 & 4136.0279 & 98.5619
\end{tabular}


Sample Name: AzO 6

Acq. Operator : SYSTEM

Sample Operator : SYSTEM

Inj : 1

Inj Volume : $5.000 \mathrm{\mu l}$

Acq. Method : Symmetry C-16 75*4.6mm,3.5mm

Mobile phase: A) $0.1 \mathrm{HCOOH}$ in $\mathrm{H} 2 \mathrm{O}$, B) $\mathrm{ACN}$

FL\low $1.0 \mathrm{ml} / \mathrm{min}$, Diluent: $\mathrm{ACN}: \mathrm{H} 20$ (93:7)

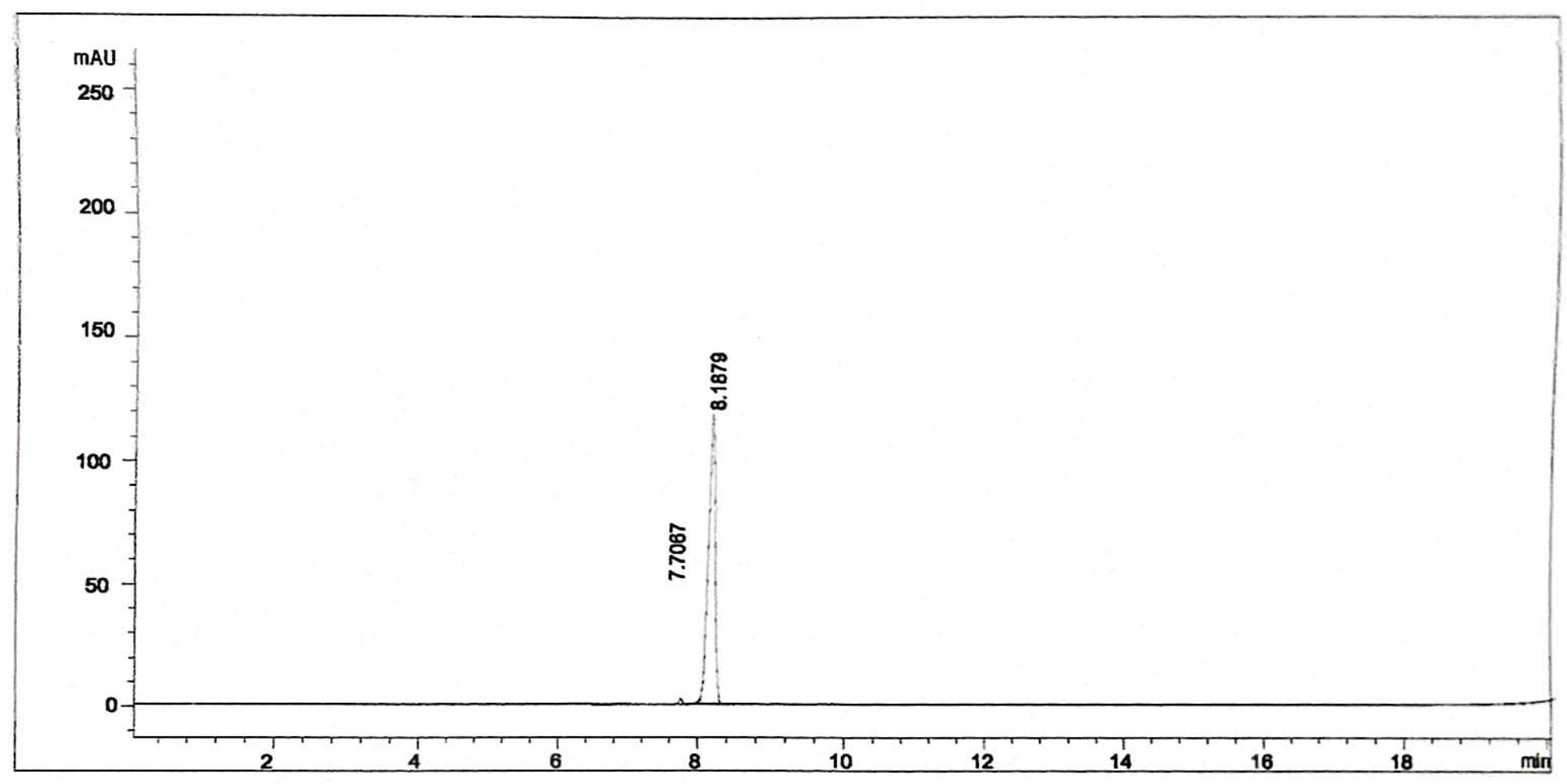

Area Percent Report

Signal 1: VWD1 A, Wavelength $=273 \mathrm{~nm}$

\begin{tabular}{|c|c|c|c|c|}
\hline $\begin{array}{c}\text { Peak } \\
\#\end{array}$ & $\begin{array}{c}\text { RetTime } \\
\text { [min] }\end{array}$ & Type & $\begin{array}{c}\text { Area } \\
{\left[\mathrm{mAU}{ }^{*} \mathrm{~s}\right]}\end{array}$ & $\begin{array}{c}\text { Area } \\
8\end{array}$ \\
\hline 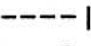 & ------ & $1-$ & & - \\
\hline 1 & 7.7067 & & 30.3719 & 0.7154 \\
\hline 2 & 8.1879 & 421 & 15.0294 & 99.2845 \\
\hline
\end{tabular}


Inj : 1

Method

: Symmetry C-16 75*4.6mm,3.5mm

Mobile phase: A) $0.1 \mathrm{HCOOH}$ in $\mathrm{H} 20$, B) $\mathrm{ACN}$

FL $\backslash$ low $1.0 \mathrm{ml} / \mathrm{min}$, Diluent: $\mathrm{ACN}: \mathrm{H} 2 \mathrm{O}$ (87:13)

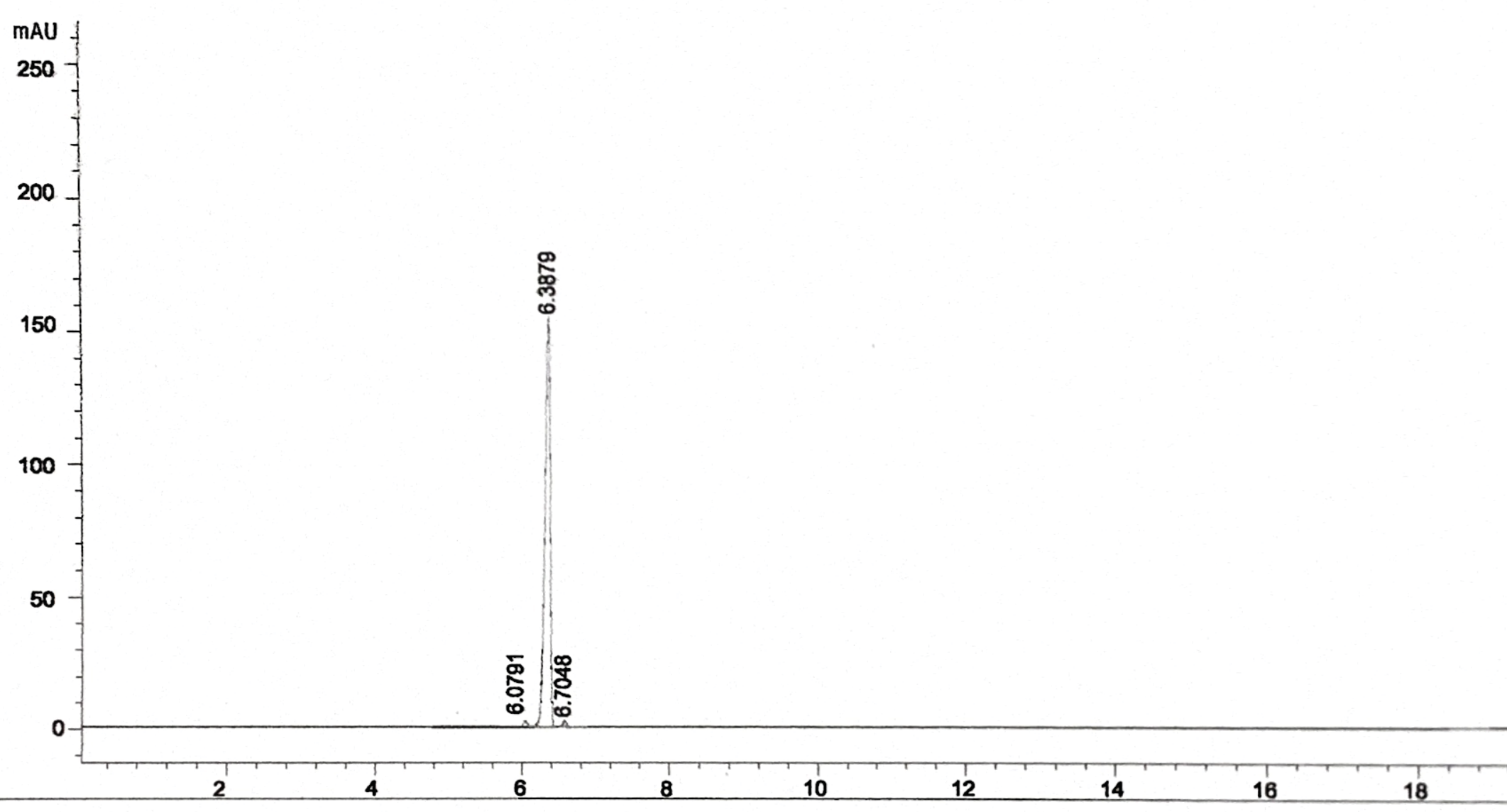

Area Percent Report

gnal 1: VWD1 A, Wavelength=273 nm

k RetTime Type Area Area

\begin{tabular}{llrr} 
& {$[\mathrm{min}]$} & {$\left[\mathrm{mAU}{ }^{*} \mathrm{~S}\right]$} & \multicolumn{1}{c}{$\frac{8}{6}$} \\
\hdashline 1 & 6.0791 & 29.0479 & 0.7018 \\
2 & 6.3879 & 4079.1976 & 98.5542 \\
3 & 6.7048 & 30.7913 & 0.7439
\end{tabular}




\section{ample Operator : SYSTEM}

Inj : 1

cq. Method

: Symmetry C-16 $75 * 4.6 \mathrm{~mm}, 3.5 \mathrm{~mm}$

Inj Volume : $5.000 \mu \mathrm{l}$

Mobile phase: A) $0.1 \mathrm{HCOOH}$ in $\mathrm{H} 2 \mathrm{O}$, B) $\mathrm{ACN}$

FL \low $1.0 \mathrm{ml} / \mathrm{min}$, Diluent: $\mathrm{ACN}: \mathrm{H} 2 \mathrm{O}$ (87:13)

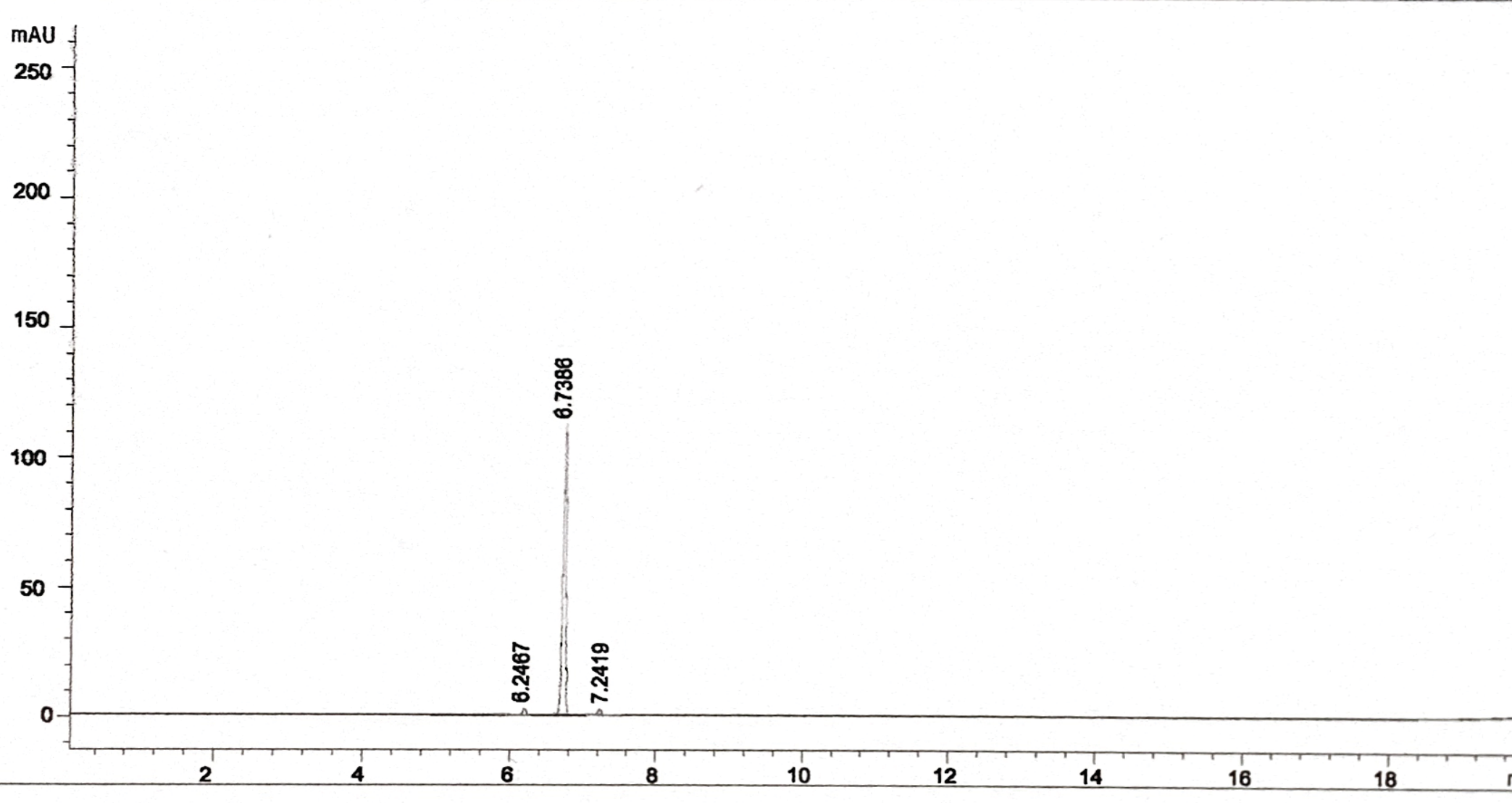

Area Percent Report

ignal 1: VWD1 A, Wavelength=273 nm

ak RetTime Type Area Area

\begin{tabular}{llcr}
$\#$ & {$[\mathrm{~min}]$} & {$\left[\mathrm{mAU} U^{*}\right]$} & \multicolumn{1}{c}{$\frac{8}{8}$} \\
\hdashline 1 & 6.2467 & 19.2376 & 0.4898 \\
2 & 6.7386 & 3883.9769 & 98.9004 \\
3 & 7.2419 & 23.9476 & 0.6097
\end{tabular}


Acq. Operator : SYSTEM
Sample Operator : SYSTEM

Inj : 1

Inj Volume : $5.000 \mathrm{\mu l}$

Acq. Method : Symmetry C-16 75*4.6mm,3.5mm

Mobile phase: A) $0.1 \mathrm{HCOOH}$ in $\mathrm{H} 20$, B) $\mathrm{ACN}$

FL \low $1.0 \mathrm{ml} / \mathrm{min}$, Diluent: $\mathrm{ACN}: \mathrm{H} 2 \mathrm{O}$ (87:13)

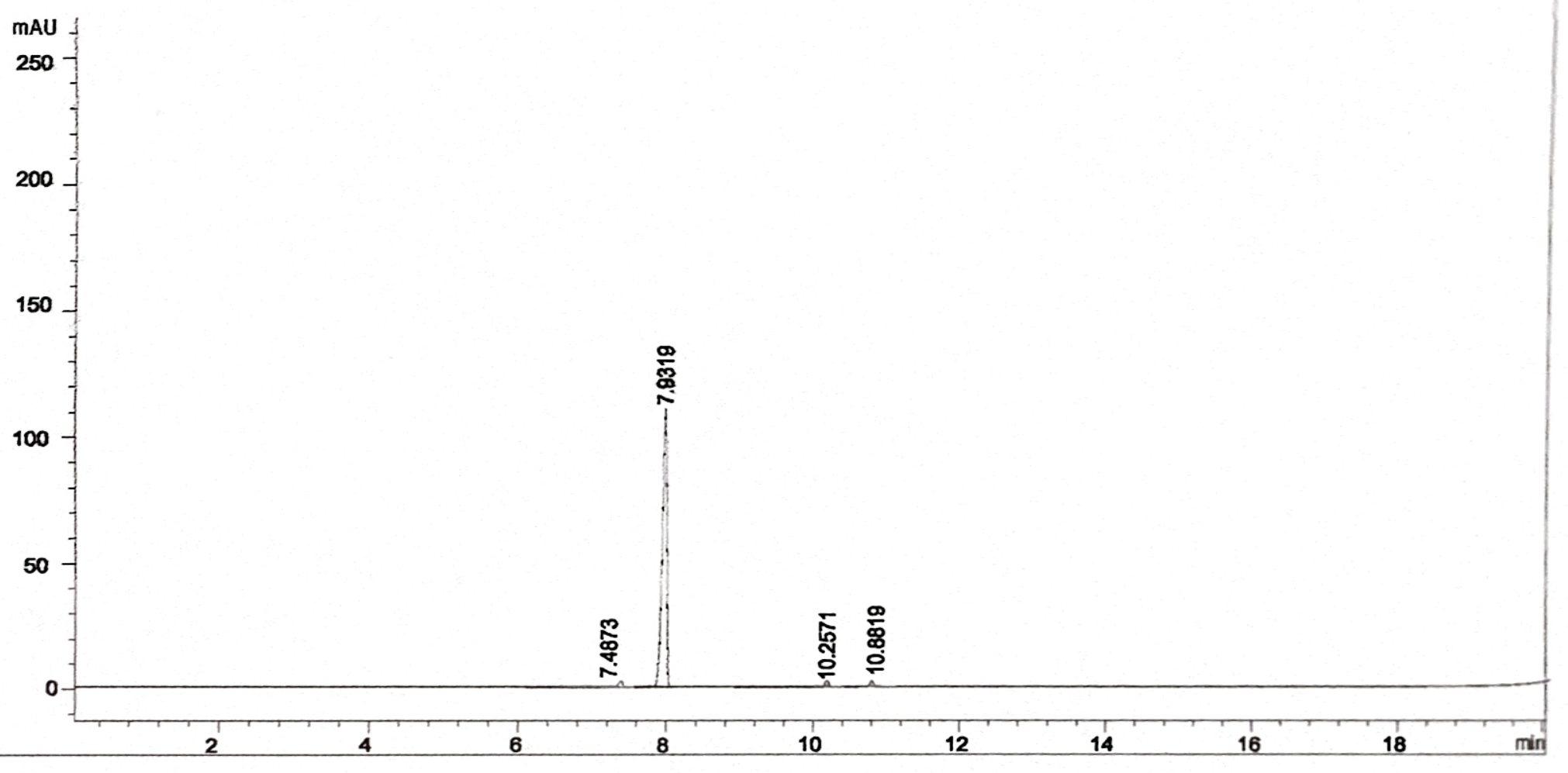

Area Percent Report

Signal 1: VWD1 A, Wavelength=273 nm

\begin{tabular}{|c|c|c|c|c|}
\hline $\begin{array}{c}\text { Peak } \\
\#\end{array}$ & $\begin{array}{c}\text { RetTime } \\
\text { [min] }\end{array}$ & Type & $\begin{array}{c}\text { Area } \\
{\left[\mathrm{mAU} U^{*} \mathrm{~s}\right]}\end{array}$ & $\begin{array}{c}\text { Area } \\
\text { \% }\end{array}$ \\
\hline- & $-\infty$ & 11 & -1 & -- \\
\hline 1 & 7.4873 & & 110.2015 & 2.5959 \\
\hline 2 & 7.9319 & & 3876.1647 & 91.3076 \\
\hline 3 & 10.2571 & & 127.8923 & 3.0126 \\
\hline 4 & 10.8819 & & 130.9137 & 3.0838 \\
\hline
\end{tabular}

Page 1 of 1 
Acq. Operator : SYSTEM

Sample Operator : SYSTEM

Inj : 1

Inj Volume : $5.000 \mu l$

Acq. Method : Symmetry C-16 $75 \star 4.6 \mathrm{~mm}, 3.5 \mathrm{~mm}$ Mobile phase: A) $0.1 \mathrm{HCOOH}$ in $\mathrm{H} 2 \mathrm{O}$, B) $\mathrm{ACN}$

FL \low $1.0 \mathrm{ml} / \mathrm{min}$, Diluent: ACN:H2O (87:13)

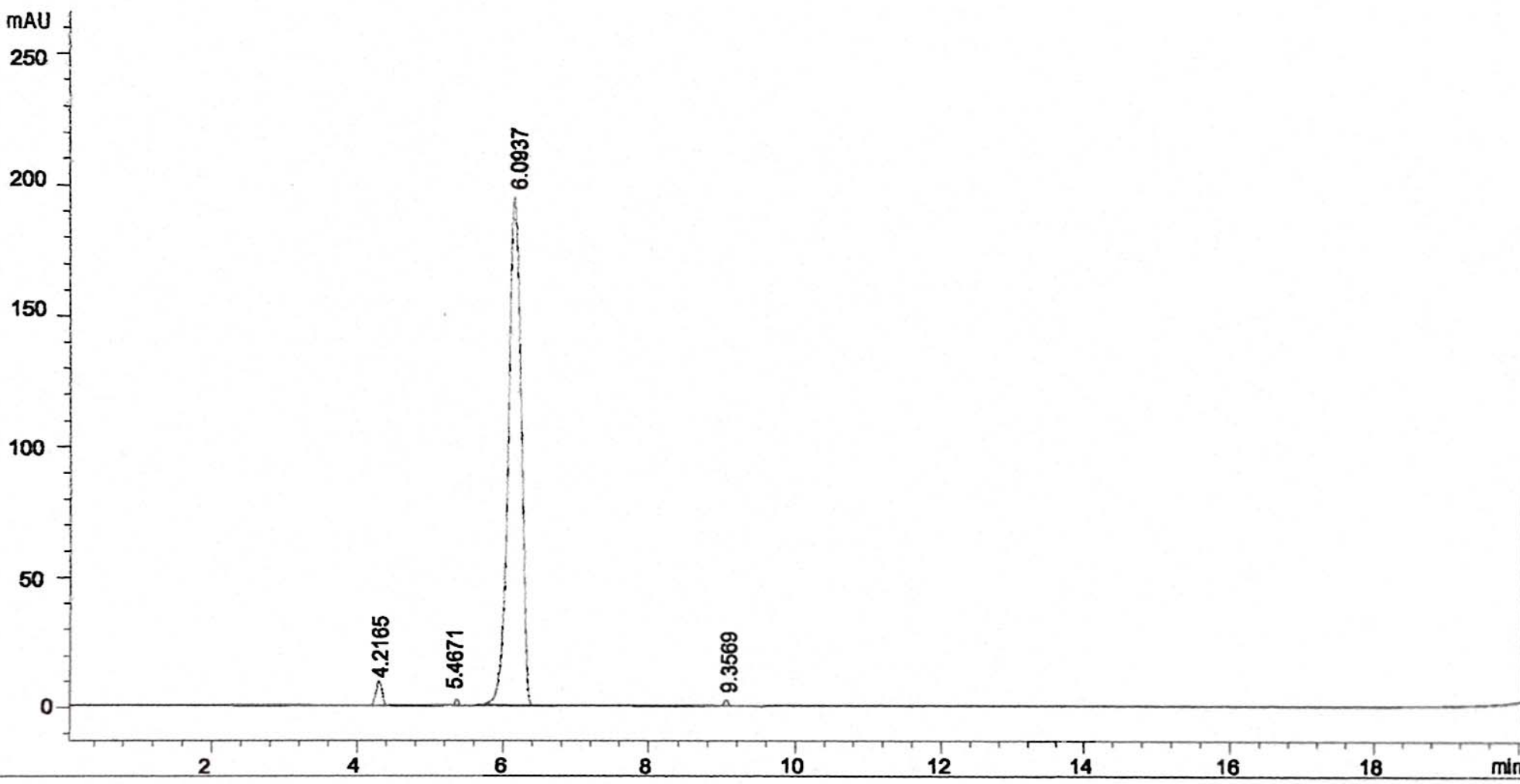

Area Percent Report

Signal 1: VWD1 A, Wavelength $=273 \mathrm{~nm}$

\begin{tabular}{|c|c|c|c|c|}
\hline $\begin{array}{c}\text { Peak } \\
\#\end{array}$ & $\begin{array}{c}\text { RetTime } \\
\text { [min] }\end{array}$ & Type & $\begin{array}{c}\text { Area } \\
{\left[\mathrm{mAU}^{*} \mathrm{~s}\right]}\end{array}$ & $\begin{array}{l}\text { Area } \\
\text { 웅 }\end{array}$ \\
\hline 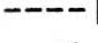 & 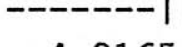 & & 500 - & $1-\cdots$ \\
\hline 1 & 4.2165 & & 583.7491 & 10.8447 \\
\hline 2 & 5.4671 & & 90.0179 & 1.6723 \\
\hline 3 & 6.0937 & & 591.7031 & 85.3033 \\
\hline 4 & 9.9768 & & 117.3284 & 2.1796 \\
\hline
\end{tabular}


Inj Volume : $5.000 \mathrm{\mu l}$

Acq. Method

: Symmetry C-16 $75 * 4.6 \mathrm{~mm}, 3.5 \mathrm{~mm}$

Mobile phase: A) $0.1 \mathrm{HCOOH}$ in $\mathrm{H} 2 \mathrm{O}$, B) $\mathrm{ACN}$

FL\low $1.0 \mathrm{ml} / \mathrm{min}$, Diluent: $\mathrm{ACN}: \mathrm{H} 2 \mathrm{O}$ (87:13)

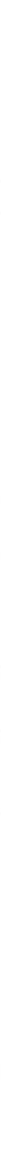

\section{Area Percent Report}

Signal 1: VWD1 A, Wavelength=273 nm

\begin{tabular}{cccc}
$\begin{array}{c}\text { Peak } \\
\#\end{array}$ & $\begin{array}{c}\text { RetTime Type } \\
\text { [min] }\end{array}$ & $\begin{array}{c}\text { Area } \\
{[\mathrm{mAU} \text { s] }}\end{array}$ & \multicolumn{1}{c}{$\begin{array}{c}\text { Area } \\
\%\end{array}$} \\
\hdashline 1 & 4.5864 & 583.6437 & 12.1076 \\
2 & 5.9713 & 75.2973 & 1.5620 \\
3 & 6.7397 & 4067.1973 & 84.3742 \\
4 & 10.1539 & 94.2973 & 1.9561
\end{tabular}


Sample Name: SP 6

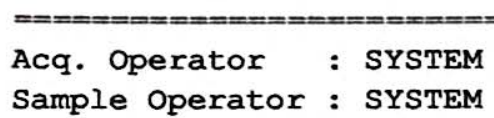

Acq. Method
: Symmetry C-16 $75 * 4.6 \mathrm{~mm}, 3.5 \mathrm{~mm}$

Mobile phase: A) $0.1 \mathrm{HCOOH}$ in $\mathrm{H} 2 \mathrm{O}$, B) $\mathrm{ACN}$

FL $\backslash$ low $1.0 \mathrm{ml} / \mathrm{min}$, Diluent: ACN:H2O (87:13)

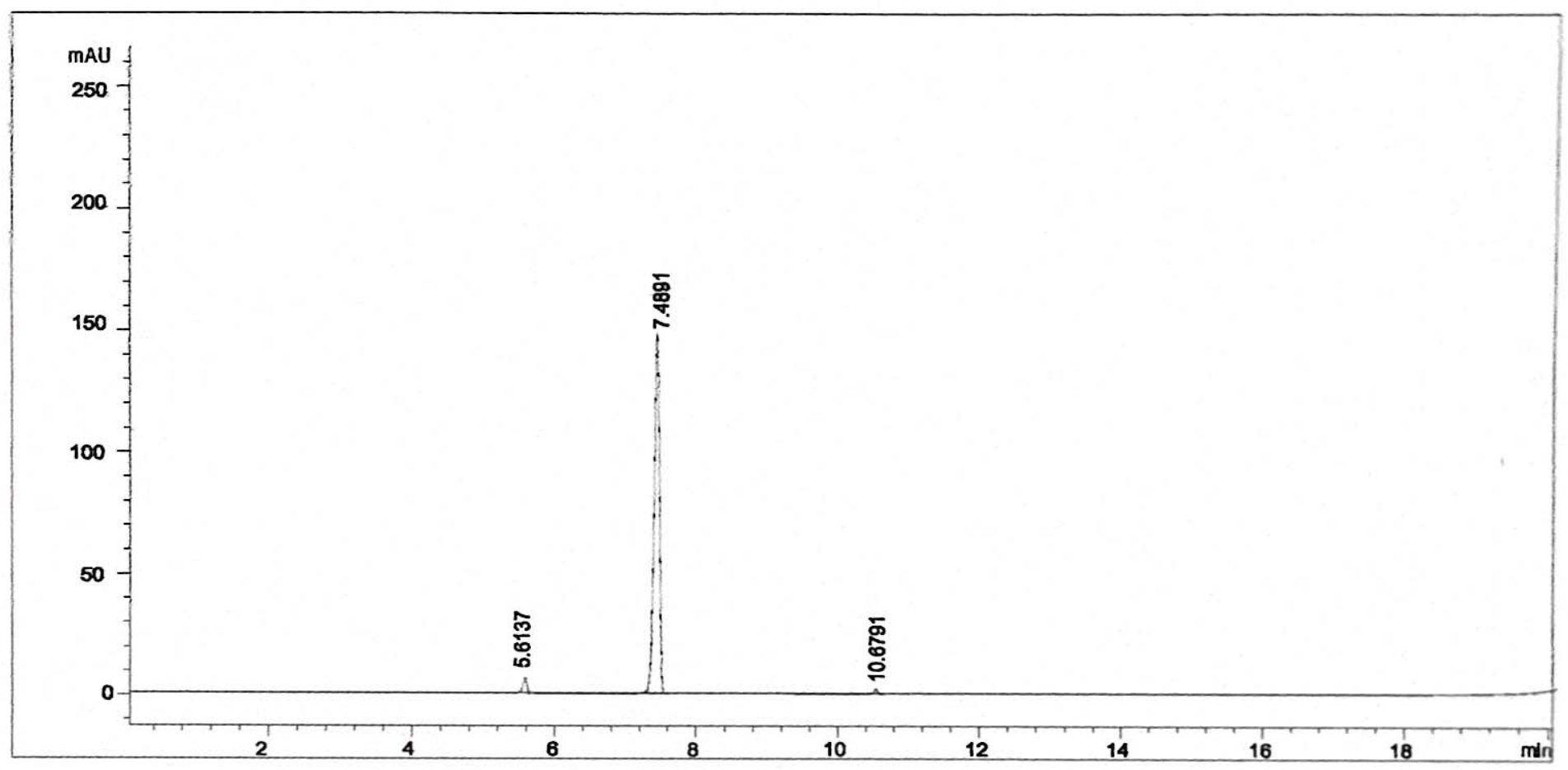

Area Percent Report

Signal 1: VWD1 A, Wavelength $=273 \mathrm{~nm}$

\begin{tabular}{|c|c|c|c|c|}
\hline $\begin{array}{c}\text { Peak } \\
\#\end{array}$ & $\begin{array}{l}\text { RetTime } \\
\text { [min] }\end{array}$ & Type & $\begin{array}{c}\text { Area } \\
{\left[\mathrm{mAU} \mathbf{U}^{\star} \mathrm{s}\right]}\end{array}$ & $\begin{array}{c}\text { Area } \\
\frac{\circ}{6}\end{array}$ \\
\hline & & & & \\
\hline 1 & 5.6137 & & 207.9437 & 5.1224 \\
\hline 2 & 7.4891 & & 3791.2379 & 93.3928 \\
\hline 3 & 10.6791 & & 60.2716 & 1.4847 \\
\hline
\end{tabular}

